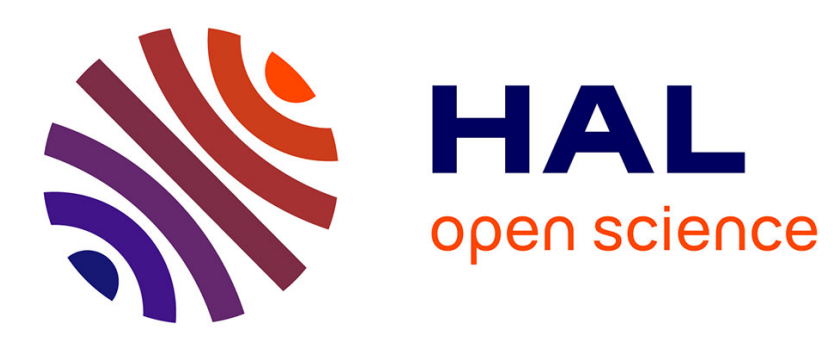

\title{
Sensor location for discrete mode observability of switching linear systems with unknown inputs
}

\author{
Taha Boukhobza
}

\section{To cite this version:}

Taha Boukhobza. Sensor location for discrete mode observability of switching linear systems with unknown inputs. Automatica, 2012, 48 (7), pp.1262-1272. 10.1016/j.automatica.2012.05.011 . hal00711319

\section{HAL Id: hal-00711319 \\ https://hal.science/hal-00711319}

Submitted on 23 Jun 2012

HAL is a multi-disciplinary open access archive for the deposit and dissemination of scientific research documents, whether they are published or not. The documents may come from teaching and research institutions in France or abroad, or from public or private research centers.
L'archive ouverte pluridisciplinaire HAL, est destinée au dépôt et à la diffusion de documents scientifiques de niveau recherche, publiés ou non, émanant des établissements d'enseignement et de recherche français ou étrangers, des laboratoires publics ou privés. 


\title{
Sensor location for discrete mode observability of switching linear systems with unknown inputs
}

\author{
T. Boukhobza \\ Centre de Recherche en Automatique de Nancy (CRAN), Nancy-University, CNRS UMR 7039 \\ email: taha.boukhobza@cran.uhp-nancy.fr
}

\begin{abstract}
This paper deals with the problem of additional sensor placement in order to recover the discrete mode observability of switching structured linear systems with unknown inputs. Such property is quite important for designing control laws, observers, fault detection and isolation schemes (when the fault occurrence implies a commutation between two modes) and so on. The proposed method, based on a graph-theoretic approach, assumes only the knowledge of the system's structure. We express, in graphical terms new necessary and sufficient conditions for the discrete mode generic observability. When these conditions are not satisfied, we propose a sensor placement procedure which allows us to recover the mode observability. Our approach can be implemented by classical and quite simple graph-theory algorithms.
\end{abstract}

Key words:

Switching linear systems, mode observability, graph theory, sensor placement.

A subtroductioybrid systems, called switching systems, is suitable to represent systems that can switch between different behaviours according to a discrete mode variable value. Many engineering systems are designed to be switching systems in order to simplify their functional description and maintain the flexibility in operation. Thus, switching systems have become ubiquitous in modern technology and the subject of significant research activities in automatic control area. For such systems, estimating the active mode is of great interest in many control areas and studies presented in (Babaali and Pappas, 2005; De Santis et al., 2009; Vidal et al., 2003) are among the most significant works which deal with this problem. In this context, our study concerns the discrete mode observability for switching linear systems (SLS) using only continuous variable measurements. More precisely two questions are addressed: establish necessary and sufficient conditions to characterise structurally the discrete mode observability and when this property is not satisfied, provide additional sensor locations in order to recover it. One motivation among others to this study lies in the fact that in many practical cases, the faults, when they occur in a system, may change completely the system dynamics. Thus, in these cases, it may be more pertinent to consider a switching linear system, where the nominal system corresponds to a specific mode and to each fault occurrence is associated a discrete mode value. Fault detectability and isolability is in this case equivalent to the discrete mode observability.

From the point of view of the class of systems under study our paper is close to (Babaali and Pappas, 2005; De Santis et al., 2006; Vidal et al., 2003; De Santis et al., 2009). Indeed, the presented work deals with the discrete mode observability of switching continuous time linear systems with unknown inputs, where the discrete mode signal is assumed to be exogenous to the continuous part and unobserved by a discrete measure. However, comparatively with the previous published results in the field, our proposed method and the obtained results present three main originalities. Firstly, few works deal with the observation of hybrid systems with unknown inputs, representing distur- bances, unmodeled dynamics or faults for example. Some studies ((Pina and Botto, 2006) and the references therein), aim at designing unknown input observers for SLS, but they do not study the mode observability conditions. Otherwise, (Vu and Liberzon, 2006) addresses the problem of recovering the discrete mode variable and the input but assumes that the initial state is known.

Secondly, most part of observability studies use algebraic or geometric approaches and so requires the exact knowledge of the state space matrices characterizing the systems' model. In many modelling problems or during conception stage, these matrices have a number of fixed zero entries determined by the physical laws while the remaining entries are not precisely known. In these cases, to study the structural properties, like observability, the idea is that we only keep the zero/non-zero entries in the state space matrices. Thus, we consider models where the fixed zeros are conserved while the non-zero entries are replaced by free parameters. Many interesting works on these models, called structured models, are related to the graph-theoretic approach and aim to analyse properties such as controllability, observability ... (Dion et al., 2003). It results from these works that the graph-theoretic approach provides quite simple and elegant analysis tool.

Finally, our approach allows us to address the problem of sensors' location to recover the discrete mode observability. This problem has not been resolved yet and is interesting from a conception point of view.

Comparatively with (Boukhobza and Hamelin, 2011) where the authors express in graphic terms necessary and sufficient conditions for the discrete mode observability of SLS, the novelties, as we highlight this after Proposition 1 , lie in the formulation of the simpler and more computationally efficient conditions. These improvements offer the possibility to propose a solution to the sensors' location problem when the discrete mode is not observable.

All the proposed results are based on classical combinatorial algorithms with polynomial order complexity. This is quite important when we deal with large scale systems. Moreover, since we consider structured systems, our ap- 
proach can be used during a conception stage.

The paper is organised as follows: after Section 2, which is devoted to the problem formulation, some definitions related to the graph-theoretic approach are given in Section 3 . The main results are stated in Section 4 before a brief conclusion.

\section{Problem statement}

Consider the following SLS

$$
\Sigma:\left\{\begin{array}{l}
\dot{x}=A(q) x+B(q) u \\
y=C(q) x+D(q) u
\end{array}\right.
$$

where $x \in \mathbb{R}^{n}, u \in \mathbb{R}^{m}$ and $y \in \mathbb{R}^{p}$ are respectively the state vector, the unknown input vector and the output (measurement) vector and where $A(\cdot), B(\cdot), C(\cdot)$ and $D(\cdot)$ are real matrices of compatible dimensions. For the exogenous and unobserved discrete mode variable (or switching signal) taking its values in $Q \stackrel{\text { def }}{=}\{1, \ldots, N\}$, we assume, as in (Babaali and Pappas, 2005), that only a finite number of jumps can occur in any finite time interval. This implies that all the system trajectories are well defined and infinitely right-differentiable. Therefore, Zeno behaviour can not occur. Without loss of generality and for the sake of homogeneity, all the inputs are assumed to be unknown. In fact, the control input signals, whose values are known, are considered to be measured i.e. we associate a virtual output equation of the form $y_{k^{\prime}}=u_{k}$ to these inputs.

Our aim is to address, in the same framework discrete mode observability of autonomous SLS and SLS with known or unknown inputs. In (De Santis et al., 2009), discrete mode observability is defined as the ability to reconstruct the mode starting from the knowledge of the input and the output, for any non-zero input value and for all initial conditions. It results that, the discrete mode observability as it is defined in (De Santis et al., 2009), cannot be used in our context, since it can never be satisfied for SLS with unknown inputs or autonomous SLS. Thus, we choose here to have a more general framework by imposing mode distinguishability not for all but for generic initial conditions $x_{0}$ and unknown inputs $u$ :

Definition 1 (Mode distinguishability) Two modes $q \in Q$ and $q^{\prime} \in Q$ with $\left(q \neq q^{\prime}\right)$ are distinguishable if at least one of the two following conditions holds:

- there exist an integer $s \geq 0$ and an expression $\Psi_{q}\left(y, \dot{y}, \ldots, y^{(s)}\right)=0$ satisfied for mode $q$ but not for mode $q^{\prime}$ for almost all initial conditions $x_{0}$ and inputs $u$. - there exist an integer $s^{\prime} \geq 0$ and an expression $\Psi_{q^{\prime}}\left(y, \dot{y}, \ldots, y^{\left(s^{\prime}\right)}\right)=0$ satisfied for mode $q^{\prime}$ but not for mode $q$ for almost all initial conditions $x_{0}$ and inputs $u$.

Here, "for almost all initial conditions $x_{0}$ and inputs $u(t)$ " is to be understood as " for all $\left(x_{0}^{T}, u^{T}\right)^{T} \in \mathbb{R}^{n+m}$ except for the zero set of some polynomials with real coefficients in the $n+m$ initial state and input components. The zero set of these polynomial forms a proper algebraic variety of $\mathbb{R}^{n+m}$ which has Lebesgue measure zero.

The interpretation of Definition 1 is that $q$ is distinguishable from $q^{\prime}$ if, for generic initial states $x_{0}$ and unknown input $u$, we can rule out $q$ or $q^{\prime}$ when observing the output over $[0, T]$.

Definition 2 (Discrete mode observability) SLS $(\Sigma)$ is discrete mode observable if its modes are all distinguishable two-by-two i.e. $\forall q \in Q, \forall q^{\prime} \in Q$, with $q \neq q^{\prime}, q$ and $q^{\prime}$ are distinguishable.

Discrete mode observability analysis can be reduced to the study of the distinguishability of each pair of modes. Thus, for the sake of simplicity, there is no loss of generality in considering that we have only two modes.

Since we study a structural property as the observability, it is pertinent to deal with structured systems, for which we assume that only the sparsity pattern of matrices $A(q)$, $B(q), C(q)$ and $D(q)$ is known for $q \in\{1,2\}$. So, to each entry of these matrices, we only know whether its value is fixed to zero, or that it has an unknown value represented by a real parameter $\lambda_{i}$. We denote by $A^{\lambda}(q), B^{\lambda}(q), C^{\lambda}(q)$ and $D^{\lambda}(q)$ respectively the matrices obtained by replacing the non-zeros in $A(q), B(q), C(q)$ and $D(q)$, for $q \in\{1,2\}$ by the corresponding parameters $\lambda_{i}$ and the studied systems have the form

$$
\Sigma_{\Lambda}:\left\{\begin{array}{l}
\dot{x}(t)=A^{\lambda}(q) x(t)+B^{\lambda}(q) u(t) \\
y(t)=C^{\lambda}(q) x(t)+D^{\lambda}(q) u(t)
\end{array}\right.
$$

If all parameters $\lambda_{i}$ are numerically fixed, we obtain a socalled admissible realization of structured switching linear systems (SSLS) $\left(\Sigma_{\Lambda}\right)$. We say that a property is true generically for SSLS $\left(\Sigma_{\Lambda}\right)$ if it is true for almost all parameters $\lambda_{i}$.

Discrete mode observability analysis is based on the distinguishability of the structured models associated to the two discrete modes. So, it is pertinent and necessary to highlight the similarities and the differences between the models associated to these modes. Thus, we decompose each structured matrix into two parts: the first one is common to the two modes and the second one is specific to each mode i.e. for $q \in\{1,2\}, A^{\lambda}(q)=A_{0}^{\lambda}+A_{q}^{\lambda}, B^{\lambda}(q)=$ $B_{0}^{\lambda}+B_{q}^{\lambda}, C^{\lambda}(q)=C_{0}^{\lambda}+C_{q}^{\lambda}$ and $D^{\lambda}(q)=D_{0}^{\lambda}+D_{q}^{\lambda}$. When we write the matrices under this form, we assume that the entries of all these matrices constituting vector $\Lambda=\left(\lambda_{1}, \lambda_{2}, \ldots, \lambda_{h}\right)^{T} \in \mathbb{R}^{h}$ can take any value in $\mathbb{R}^{h}$ or equivalently that parameters $\lambda_{i}$ are free. Note that there is no third mode 0 but we use $q=0$ as subscript to represent the common part between the two modes, in order to exhibit structurally their differences and their similarities. All the results exposed in the paper lean on the hypothesis that all the entries of matrices $A_{0}^{\lambda}, A_{q}^{\lambda}, B_{0}^{\lambda}, B_{q}^{\lambda}, C_{0}^{\lambda}$, $C_{q}^{\lambda}, D_{0}^{\lambda}$ and $D_{q}^{\lambda}$ are free. This can be obtained when dealing with a model close to the physical laws governing the system. On the one hand, for any other decomposition of $A^{\lambda}(q), B^{\lambda}(q), C^{\lambda}(q)$ and $D^{\lambda}(q)$ which does not satisfy this hypothesis, the method presented here is not guaranteed. On the other hand, when applying a coordinate change, if we break the parameter independence or in other terms if we obtain matrices having entries linked by fixed algebraic relations, then the results of the paper are not guaranteed. Note that by not guaranteed, we mean that the results may be correct may be not. It depends if the relation between the parameters introduce a singularity in the different structural properties used in the proposed method (observability subspace dimensions for example).

To summarize, our aim is to provide necessary and sufficient conditions to check if a SSLS is generically discrete mode observable i.e. is discrete mode observable for almost 
all its realizations or equivalently for almost all parameters $\lambda_{i}$. Next, when these conditions are not satisfied, we provide a procedure for adding sensors in order to recover one of them and so the discrete mode observability property. Note that we do not address, as in (Boukhobza and Hamelin, 2011), the problem of state estimation of SLS in the presence of unknown inputs.

\section{Graphical representation and definitions}

The digraph associated to $\left(\Sigma_{\Lambda}\right)$ is noted $\mathcal{G}\left(\Sigma_{\Lambda}\right)$. It is constituted by a vertex set $\mathcal{V}$ and an edge set $\mathcal{E}$ i.e. $\mathcal{G}\left(\Sigma_{\Lambda}\right)=(\mathcal{V}, \mathcal{E})$. The vertices are associated to the continuous state, the input and the output components of $\left(\Sigma_{\Lambda}\right)$ and the directed edges represent links between these variables. More precisely, $\mathcal{V}=\mathbf{X} \cup \mathbf{U} \cup \mathbf{Y}$, where $\mathbf{X}=\left\{\mathbf{x}_{\mathbf{1}}, \ldots, \mathbf{x}_{\mathbf{n}}\right\}, \mathbf{U}=\left\{\mathbf{u}_{\mathbf{1}}, \ldots, \mathbf{u}_{\mathbf{m}}\right\}$ and $\mathbf{Y}=\left\{\mathbf{y}_{1}, \ldots, \mathbf{y}_{\mathbf{p}}\right\}$ are respectively the set of state, input and output vertices. For $q \in\{0,1,2\}$, we define $\mathcal{E}_{q}=A_{q}$-edges $\cup B_{q}$-edges $\cup C_{q}$-edges $\cup D_{q}$-edges, where, for $q \in\{0,1,2\}, A_{q}$-edges $=\left\{\left(\mathbf{x}_{\mathbf{j}}, \mathbf{x}_{\mathbf{i}}\right) \mid A_{q}(i, j) \neq 0\right\}$, $B_{q}$-edges $=\left\{\left(\mathbf{u}_{\mathbf{j}}, \mathbf{x}_{\mathbf{i}}\right) \mid B_{q}(i, j) \neq 0\right\}, C_{q}$-edges $=$ $\left\{\left(\mathbf{x}_{\mathbf{j}}, \mathbf{y}_{\mathbf{i}}\right) \mid C_{q}(i, j) \neq 0\right\} \quad$ and $D_{q}$-edges $=$ $\left\{\left(\mathbf{u}_{\mathbf{j}}, \mathbf{y}_{\mathbf{i}}\right) \mid D_{q}(i, j) \neq 0\right\}$. Finally the edge set is $\mathcal{E}=\bigcup_{q=0}^{2} \mathcal{E}_{q}$. Here, $M(i, j)$ is the $(i, j)$ th element of matrix $M$ and $\left(\mathbf{v}_{\mathbf{1}}, \mathbf{v}_{\mathbf{2}}\right)$ denotes a directed edge from vertex $\mathbf{v}_{\mathbf{1}} \in \mathcal{V}$ to vertex $\mathbf{v}_{\mathbf{2}} \in \mathcal{V}$. The edges included in $\mathcal{E}_{0}$ represent the common part of the two modes' models, while $\mathcal{E}_{q}$, for $q \in\{1,2\}$ is related to the specific part of each mode's model. For the sake of clarity, the vertices are written in bold fonts to differentiate them from the corresponding variables. Each edge is associated to a free non-zero parameter of the system's model called the weight of the edge. Note that number $q$ is written under each $\mathcal{E}_{q}$-edge and represents its index. An edge belonging to $\mathcal{E}_{q}, q \neq 0$, is said specific to mode $q$. Note that even if it is conceptually feasible to have an edge with index 0 having the same end vertices than an edge with index $q \neq 0$, we will, for a sake of coherence and readability, avoid this case and prefer two edges with indices 1 and 2 . The index 0 will, then be reserved to the common part of the two modes.

Example 1 To the system defined by the matrices below, we associate the digraph in Figure 1.

$\begin{aligned} A_{0}^{\lambda} & =\left(\begin{array}{cccccccc}0 & 0 & 0 & 0 & 0 & 0 & 0 & 0 \\ 0 & 0 & \lambda_{1} & 0 & 0 & 0 & 0 & 0 \\ 0 & \lambda_{2} & 0 & 0 & 0 & 0 & 0 & 0 \\ 0 & 0 & 0 & 0 & 0 & 0 & 0 & \lambda_{3} \\ 0 & 0 & 0 & 0 & 0 & 0 & 0 & 0 \\ 0 & 0 & 0 & 0 & 0 & 0 & 0 & 0 \\ 0 & 0 & 0 & 0 & \lambda_{4} & 0 & 0 & 0 \\ 0 & 0 & 0 & 0 & 0 & 0 & \lambda_{5} & 0\end{array}\right), B_{0}^{\lambda}=\left(\begin{array}{c}0 \\ 0 \\ 0 \\ 0 \\ 0 \\ \lambda_{6} \\ 0 \\ 0\end{array}\right), B_{1}^{\lambda}=B_{2}^{\lambda}= \\ 0, C_{0}^{\lambda}= & \left(\begin{array}{cccccccc}\lambda_{7} & 0 & 0 & 0 & 0 & 0 & 0 & 0 \\ 0 & 0 & 0 & 0 & \lambda_{8} & 0 & 0 & 0 \\ 0 & 0 & 0 & \lambda_{9} & 0 & 0 & 0 & 0 \\ 0 & 0 & 0 & 0 & 0 & 0 & 0 & \lambda_{10}\end{array}\right) \text {, all the entries of } A_{1}^{\lambda} \text { are }\end{aligned}$ zero except $A_{1}^{\lambda}(1,2)=\lambda_{11}, A_{1}^{\lambda}(4,4)=\lambda_{12}, A_{1}^{\lambda}(4,5)=$ $\lambda_{13}, A_{1}^{\lambda}(5,2)=\lambda_{14}$ and $A_{1}^{\lambda}(7,6)=\lambda_{15}$ all the entries of
$A_{2}^{\lambda}$ are zero except $A_{2}^{\lambda}(4,5)=\lambda_{16}, A_{2}^{\lambda}(5,2)=\lambda_{17}$ and $A_{2}^{\lambda}(7,6)=\lambda_{18}$, the elements of matrices $C_{1}^{\lambda}, C_{2}^{\lambda}, D_{0}^{\lambda}, D_{1}^{\lambda}$ and $D_{2}^{\lambda}$ are equal to zero.

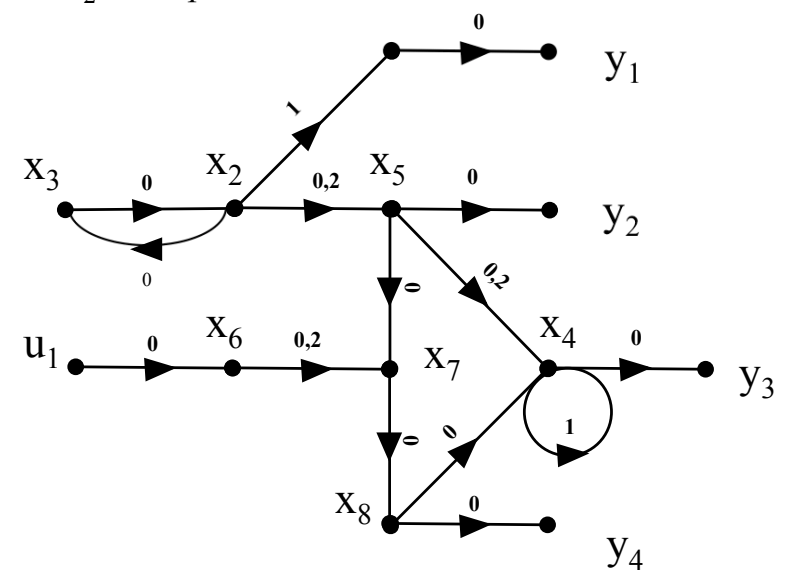

Figure 1. Digraph associated to system of Example 1 A realisation of this system could be the numerical specified system having the following dynamics:

Mode $1:\left\{\begin{array}{l}\dot{x}_{1}=x_{2} \\ \dot{x}_{2}=-x_{3} \\ \dot{x}_{3}=2 x_{2} \\ \dot{x}_{4}=-3 x_{4}+x_{5}-x_{8} \\ \dot{x}_{5}=2 x_{2} \\ \dot{x}_{6}=u_{1} \\ \dot{x}_{7}=-2 x_{5}+x_{6} \\ \dot{x}_{8}=2 x_{7} \\ y_{1}=x_{1} \\ y_{2}=x_{5} \\ y_{3}=x_{4} \\ y_{4}=x_{8}\end{array} \quad\right.$ Mode $2:\left\{\begin{array}{l}\dot{x}_{1}=0 \\ \dot{x}_{2}=-x_{3} \\ \dot{x}_{3}=2 x_{2} \\ \dot{x}_{4}=2 x_{5}-x_{8} \\ \dot{x}_{5}=-x_{2} \\ \dot{x}_{6}=u_{1} \\ \dot{x}_{7}=-2 x_{5}+3 x_{6} \\ \dot{x}_{8}=2 x_{7} \\ y_{1}=x_{1} \\ y_{2}=x_{5} \\ y_{3}=x_{4} \\ y_{4}=x_{8}\end{array}\right.$

The digraph representing the SSLS is built from the superposition of the digraphs related to each mode. In order to study the properties of the system associated to a specific mode $q$, we have to restrict the edge set to $\mathcal{E}_{0} \cup \mathcal{E}_{q}$. In this context, many of the functions and specific vertex subsets, defined below, present an index $q$ related to the considered mode.

Let us now give some useful definitions and notations.

- A path is simple when every vertex occurs only once in this path.

- A cycle is a path of the form $\mathbf{v}_{\mathbf{s}_{0}} \rightarrow \mathbf{v}_{\mathbf{s}_{1}} \rightarrow \ldots \rightarrow$ $\mathbf{v}_{\mathbf{s}_{\mathbf{i}}} \rightarrow \mathbf{v}_{\mathbf{s}_{\mathbf{0}}}$, where $\mathbf{v}_{\mathbf{s}_{\mathbf{0}}}, \mathbf{v}_{\mathbf{s}_{\mathbf{1}}}, \ldots, \mathbf{v}_{\mathbf{s}_{\mathbf{i}}}$ are distinct.

- For $q \in\{1,2\}$, we say that path $P$ is included in $\mathcal{E}_{0} \cup \mathcal{E}_{q}$ if all its edges are included in $\mathcal{E}_{0} \cup \mathcal{E}_{q}$.

- A path is a $\mathbf{Y}$-topped path if its end vertex belongs to $\mathbf{Y}$. In the sequel, $\mathcal{V}_{1}$ and $\mathcal{V}_{2}$ represent two subsets of $\mathcal{V}$. We denote by $\operatorname{card}(\cdot)$ the cardinality function and $\mathcal{V}_{1} \backslash \mathcal{V}_{2}$ is the set of elements in $\mathcal{V}_{1}$ which are not in $\mathcal{V}_{2}$.

- Denote by $\operatorname{Pred}_{q}\left(\mathcal{V}_{1}\right)$ the set of all the predecessors of vertices included in $\mathcal{V}_{1}$ in mode $q$.

- A path $P=\mathbf{v}_{\mathbf{s}_{\mathbf{0}}} \rightarrow \mathbf{v}_{\mathbf{s}_{1}} \rightarrow \ldots \rightarrow \mathbf{v}_{\mathbf{s}_{\mathbf{i}}}$ is said a $\mathcal{V}_{1} \rightarrow \mathcal{V}_{2}$ path if $\mathbf{v}_{\mathbf{s}_{\mathbf{o}}} \in \mathcal{V}_{1}$ and $\mathbf{v}_{\mathbf{s}_{\mathbf{i}}} \in \mathcal{V}_{2}$. Moreover, if the only vertex of $P$ which belongs to $\mathcal{V}_{1}$ is $\mathbf{v}_{\mathbf{s}_{0}}$ and the only vertex of $P$ which belongs to $\mathcal{V}_{2}$ is $\mathbf{v}_{\mathbf{s}_{\mathbf{i}}}, P$ is called a direct $\mathcal{V}_{1} \rightarrow \mathcal{V}_{2}$ 
path.

- For $q=\{1,2\}, \rho_{q}\left[\mathcal{V}_{1}, \mathcal{V}_{2}\right]$ is the maximal number of disjoint $\mathcal{V}_{1} \rightarrow \mathcal{V}_{2}$ paths included in $\mathcal{E}_{0} \cup \mathcal{E}_{q}$. Moreover, a set of $\rho_{q}\left[\mathcal{V}_{1}, \mathcal{V}_{2}\right]$ disjoint $\mathcal{V}_{1} \rightarrow \mathcal{V}_{2}$ paths included in $\mathcal{E}_{0} \cup \mathcal{E}_{q}$ is called maximum $\mathcal{V}_{1} \rightarrow \mathcal{V}_{2}$ linking in $\mathcal{E}_{0} \cup \mathcal{E}_{q}$.

- For $q \in\{1,2\}, \mu_{q}\left[\mathcal{V}_{1}, \mathcal{V}_{2}\right]$ denotes the minimal number of vertices of $\mathbf{U} \cup \mathbf{X} \cup \mathbf{Y}$ belonging to a maximum $\mathcal{V}_{1} \rightarrow \mathcal{V}_{2}$ linking included in $\mathcal{E}_{0} \cup \mathcal{E}_{q}$.

- For $q \in\{1,2\}, V_{e s s, q}\left[\mathcal{V}_{1}, \mathcal{V}_{2}\right]$ is the vertex subset including the vertices present in all the maximum $\mathcal{V}_{1} \rightarrow \mathcal{V}_{2}$ linkings included in $\mathcal{E}_{0} \cup \mathcal{E}_{q}$.

- For $q \in\{1,2\}$, there exists a unique vertex subset noted $S_{q}^{o}\left[\mathcal{V}_{1}, \mathcal{V}_{2}\right]$ and called minimum output separator which is the set of begin vertices of all direct $V_{\text {ess }, q}\left[\mathcal{V}_{1}, \mathcal{V}_{2}\right] \rightarrow \mathcal{V}_{2}$ paths included in $\mathcal{E}_{0} \cup \mathcal{E}_{q}$.

- For $q \in\{1,2\}$, there exists a unique vertex subset noted $S_{q}^{i}\left[\mathcal{V}_{1}, \mathcal{V}_{2}\right]$ and called minimum input separator which is the set of end vertices of all direct $\mathcal{V}_{1} \rightarrow V_{e s s, q}\left[\mathcal{V}_{1}, \mathcal{V}_{2}\right]$ paths included in $\mathcal{E}_{0} \cup \mathcal{E}_{q}$.

In Example $1, \rho_{1}[\mathbf{U}, \mathbf{Y}]=\rho_{2}[\mathbf{U}, \mathbf{Y}]=1$ and $\rho_{1}\left[\left\{\mathbf{x}_{1}, \mathbf{x}_{\mathbf{2}}, \mathbf{x}_{\mathbf{3}}\right\}, \mathbf{Y}\right]=\rho_{2}\left[\left\{\mathbf{x}_{1}, \mathbf{x}_{\mathbf{2}}, \mathbf{x}_{\mathbf{3}}\right\}, \mathbf{Y}\right]=$ 2. Moreover, $V_{\text {ess }, 2}\left[\left\{\mathbf{x}_{\mathbf{3}}\right\}, \mathbf{Y}\right]=\left\{\mathbf{x}_{\mathbf{3}}, \mathbf{x}_{\mathbf{2}}, \mathbf{x}_{\mathbf{5}}\right\}$ but $V_{\text {ess }, 1}\left[\left\{\mathbf{x}_{\mathbf{3}}\right\}, \mathbf{Y}\right]=\left\{\mathbf{x}_{\mathbf{3}}, \mathbf{x}_{\mathbf{2}}\right\}$ because there exists in $\mathcal{E}_{0} \cup$ $\mathcal{E}_{1}$ a $\mathbf{x}_{3} \rightarrow \mathbf{Y}$ path which does not cover $\mathbf{x}_{\mathbf{5}}: \mathbf{x}_{\mathbf{3}} \rightarrow \mathbf{x}_{\mathbf{2}} \rightarrow$ $\mathbf{x}_{\mathbf{1}} \rightarrow \mathbf{y}_{\mathbf{1}}$. Finally, $S_{1}^{o}[\mathbf{U}, \mathbf{Y}]=\left\{\mathbf{x}_{\mathbf{8}}\right\}, S_{2}^{o}\left[\left\{\mathbf{x}_{\mathbf{3}}\right\}, \mathbf{Y}\right]=$ $\left\{\mathbf{x}_{\mathbf{5}}\right\}, S_{1}^{o}\left[\left\{\mathbf{x}_{\mathbf{2}}, \mathbf{x}_{\mathbf{3}}\right\}, \mathbf{Y}\right]=\left\{\mathbf{x}_{\mathbf{2}}\right\}, S_{2}^{o}\left[\left\{\mathbf{x}_{\mathbf{2}}, \mathbf{x}_{\mathbf{3}}\right\}, \mathbf{Y}\right]=$ $\left\{\mathbf{x}_{\mathbf{5}}\right\}, S_{1}^{i}\left[\left\{\mathbf{x}_{\mathbf{2}}, \mathbf{x}_{\mathbf{3}}\right\}, \mathbf{Y}\right]=\left\{\mathbf{x}_{\mathbf{2}}\right\}=S_{2}^{i}\left[\left\{\mathbf{x}_{\mathbf{2}}, \mathbf{x}_{\mathbf{3}}\right\}, \mathbf{Y}\right]$.

\section{Main result}

\subsection{Preliminaries}

Let $\mathbf{V}_{\mathbf{Y}} \subseteq \mathbf{Y}$ denotes a vertex subset playing the role of the output vertex subset instead of $\mathbf{Y}$. Our aim is to characterize the generic dimension of the observability subspace when only measurements related to $\mathbf{V}_{\mathbf{Y}}$ are available. To do so, we subdivide first the system into two parts from the observability point of view: the maximal over-determined part and the rest related to the square part and the underdetermined part. The discrete mode observability can be achieved only in the over-determined part. This subdivision is provided in (van der Woude, 2000) and is equivalent to the Dulmage-Mendelsohn decomposition when representing the system by a bipartite graph.

Definition 3 (Boukhobza et al., 2007) In digraph $\mathcal{G}\left(\Sigma_{\Lambda}\right)$, for each vertex subset $\mathbf{V}_{\mathbf{Y}} \subseteq \mathbf{Y}$ and $q \in\{1,2\}$, we define: $\bullet \mathbf{X}_{\mathbf{1}, \mathbf{q}}\left(\mathbf{V}_{\mathbf{Y}}\right) \stackrel{\text { def }}{=}$ $\left\{\mathbf{x}_{\mathbf{i}} \mid \rho_{q}\left[\mathbf{U} \cup\left\{\mathbf{x}_{\mathbf{i}}\right\}, \mathbf{V}_{\mathbf{Y}}\right]>\rho_{q}\left[\mathbf{U}, \mathbf{V}_{\mathbf{Y}}\right]\right\}$

- $\mathbf{Y}_{\mathbf{0}, \mathbf{q}}\left(\mathbf{V}_{\mathbf{Y}}\right) \stackrel{\text { def }}{=} \mathbf{V}_{\mathbf{Y}} \cap V_{\text {ess }, q}\left[\mathbf{U}, \mathbf{V}_{\mathbf{Y}}\right]$;

- $\mathbf{Y}_{\mathbf{1}, \mathbf{q}}\left(\mathbf{V}_{\mathbf{Y}}\right) \stackrel{\text { def }}{=} \mathbf{V}_{\mathbf{Y}} \backslash \mathbf{Y}_{\mathbf{0 , q}}\left(\mathbf{V}_{\mathbf{Y}}\right)$;

- $\mathbf{U}_{\mathbf{0}, \mathbf{q}}\left(\mathbf{V}_{\mathbf{Y}}\right) \stackrel{\text { def }}{=}\left\{\mathbf{u}_{\mathbf{i}} \mid\right.$ there is no edge from $\mathbf{u}_{\mathbf{i}}$ to $\left.\mathbf{X}_{\mathbf{1}, \mathbf{q}}\left(\mathbf{V}_{\mathbf{Y}}\right) \cup \mathbf{Y}_{\mathbf{1}, \mathbf{q}}\left(\mathbf{V}_{\mathbf{Y}}\right)\right\}$;

- $\mathbf{U}_{\mathbf{1}, \mathbf{q}}\left(\mathbf{V}_{\mathbf{Y}}\right) \stackrel{\text { def }}{=} \mathbf{U} \backslash \mathbf{U}_{\mathbf{0}, \mathbf{q}}\left(\mathbf{V}_{\mathbf{Y}}\right)$;

- $\mathbf{X}_{\mathbf{s}, \mathbf{q}}\left(\mathbf{V}_{\mathbf{Y}}\right) \stackrel{\text { def }}{=} S_{q}^{o}\left[\mathbf{U}_{\mathbf{0}, \mathbf{q}}\left(\mathbf{V}_{\mathbf{Y}}\right), \mathbf{V}_{\mathbf{Y}}\right] \cap \mathbf{X}$;

- $\mathbf{X}_{\mathbf{0}, \mathbf{q}}\left(\mathbf{V}_{\mathbf{Y}}\right) \stackrel{\text { def }}{=} \mathbf{X} \backslash\left(\mathbf{X}_{\mathbf{1}, \mathbf{q}}\left(\mathbf{V}_{\mathbf{Y}}\right) \cup \mathbf{X}_{\mathbf{s}, \mathbf{q}}\left(\mathbf{V}_{\mathbf{Y}}\right)\right)$.

We can uniquely decompose for each mode the system into two subsystems:

- $\left(\Sigma_{1, q}\right)$, representing, from the observability point of view, the over-determined part of the system. Indeed, it is proven in (Boukhobza et al., 2007; van der Woude, 2000) that this part remains left invertible even if we remove any one of its measurements. This is due to the fact that, in this part, by definition no output vertex is essential in an input-output linking and all the state vertices constituting this subsystem can reach an output vertex independently from the input-output paths. It has necessarily more outputs (measurements) than inputs and is defined by input $\mathbf{U}_{\mathbf{1}, \mathbf{q}}\left(\mathbf{V}_{\mathbf{Y}}\right)$ and $\mathbf{X}_{\mathbf{s}, \mathbf{q}}\left(\mathbf{V}_{\mathbf{Y}}\right)$, state $\mathbf{X}_{\mathbf{1}, \mathbf{q}}\left(\mathbf{V}_{\mathbf{Y}}\right)$ and output $\mathbf{Y}_{\mathbf{1}, \mathbf{q}}\left(\mathbf{V}_{\mathbf{Y}}\right)$.

- $\left(\Sigma_{0, q}\right)$ defined by input $\mathbf{U}_{\mathbf{0}, \mathbf{q}}\left(\mathbf{V}_{\mathbf{Y}}\right)$, state $\mathbf{X}_{\mathbf{0}, \mathbf{q}}\left(\mathbf{V}_{\mathbf{Y}}\right)$ and output $\mathbf{X}_{\mathbf{s}, \mathbf{q}}\left(\mathbf{V}_{\mathbf{Y}}\right) \cup \mathbf{Y}_{\mathbf{0 , q}}\left(\mathbf{V}_{\mathbf{Y}}\right)$. This subsystem represents the square and under-determined parts of the system.

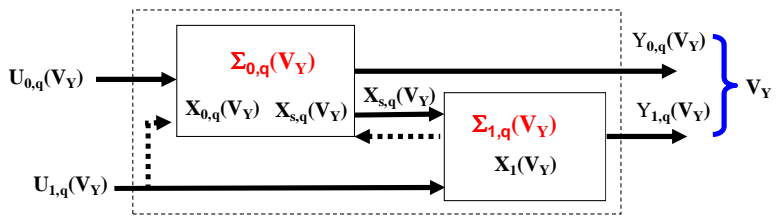

Figure 2. System subdivision

In $\quad$ Example $1, \mathbf{X}_{1, \mathbf{1}}(\mathbf{Y}) \quad$ $\left\{\mathbf{x}_{1}, \mathbf{x}_{2}, \mathbf{x}_{3}, \mathbf{x}_{4}, \mathbf{x}_{5}\right\}, \mathbf{Y}_{1,1}(\mathbf{Y})=\mathbf{Y}_{\mathbf{1}}, \mathbf{Y}_{0,1}(\mathbf{Y})=$
$\emptyset=\mathbf{U}_{1,1}(\mathbf{Y}), \mathbf{U}_{0,1}(\mathbf{Y})=\left\{\mathbf{u}_{1}\right\}, \mathbf{X}_{\mathbf{s}, \mathbf{1}}(\mathbf{Y})=\left\{\mathbf{x}_{\mathbf{8}}\right\}$, $\mathbf{X}_{\mathbf{0 , 1}}(\mathbf{Y})=\left\{\mathbf{x}_{\mathbf{6}}, \mathbf{x}_{\mathbf{7}}\right\}$. The decomposition is the same for $q=2$.

For $\mathbf{V}_{\mathbf{Y}}=\mathbf{Y}$ and for each $q \in\{1,2\}, X_{0, q}, X_{s, q}, X_{1, q}$, $U_{0, q}, U_{1, q}, Y_{0, q}$ and $Y_{1, q}$ denote the state, the unknown input and the output associated to vertex subsets $\mathbf{X}_{\mathbf{0}, \mathbf{q}}(\mathbf{Y})$, $\mathbf{X}_{\mathbf{s}, \mathbf{q}}(\mathbf{Y}), \mathbf{X}_{\mathbf{1}, \mathbf{q}}(\mathbf{Y}), \mathbf{U}_{\mathbf{0}, \mathbf{q}}(\mathbf{Y}), \mathbf{U}_{\mathbf{1}, \mathbf{q}}(\mathbf{Y}), \mathbf{Y}_{\mathbf{0}, \mathbf{q}}(\mathbf{Y})$ and $\mathbf{Y}_{\mathbf{1}, \mathbf{q}}(\mathbf{Y})$ vectors respectively.

Remark 1 When all the inputs are known, or equivalently measured, then subsystem $\Sigma_{0, q}, q \in 1,2$ contains only the state vertices which are not connected to the measurements.

Important remarks are summarized in the following lemmas:

Lemma 1 There cannot exist a relation linking only the output components of square subsystem $\Sigma_{0, q}, Y_{0, q}$ and $X_{s, q}$.

Proof: Using results shown in (van der Woude, 2000) (Theorem 5.1) concerning the case of square systems, we have that the generic number of invariant zeros of the pencil matrix of system $\Sigma_{0, q}$ is equal to $\operatorname{card}\left(\mathbf{X}_{\mathbf{0}, \mathbf{q}}\right)+\operatorname{card}\left(\mathbf{X}_{\mathbf{s}, \mathbf{q}}\right)+$ $\operatorname{card}\left(\mathbf{U}_{\mathbf{0}, \mathbf{q}}\right)-\left(\mu_{q}\left[\mathbf{U}_{\mathbf{0}, \mathbf{q}}(\mathbf{Y}), \mathbf{X}_{\mathbf{s}, \mathbf{q}}(\mathbf{Y}) \cup \mathbf{Y}_{\mathbf{0}, \mathbf{q}}(\mathbf{Y})\right]-\right.$ $\left.\rho_{q}\left[\mathbf{U}_{\mathbf{0}, \mathbf{q}}(\mathbf{Y}), \mathbf{X}_{\mathbf{s}, \mathbf{q}}(\mathbf{Y}) \cup \mathbf{Y}_{\mathbf{0}, \mathbf{q}}(\mathbf{Y})\right]+\operatorname{card}\left(\mathbf{X}_{\mathbf{s}, \mathbf{q}}\right)\right)$.

Thus, the dimension of the strongly observable subspace and so the number of possible independent observation equations is equal to $\mu_{q}\left[\mathbf{U}_{\mathbf{0}, \mathbf{q}}(\mathbf{Y}), \mathbf{X}_{\mathbf{s}, \mathbf{q}}(\mathbf{Y}) \cup\right.$ $\left.\mathbf{Y}_{\mathbf{0}, \mathbf{q}}(\mathbf{Y})\right]-\rho_{q}\left[\mathbf{U}_{\mathbf{0}, \mathbf{q}}(\mathbf{Y}), \mathbf{X}_{\mathbf{s}, \mathbf{q}}(\mathbf{Y}) \cup \mathbf{Y}_{\mathbf{0}, \mathbf{q}}(\mathbf{Y})\right]$. Moreover, the cardinality of $\mathbf{U}_{\mathbf{0 , q}}(\mathbf{Y}) \cup \mathbf{X}_{\mathbf{0}, \mathbf{q}}(\mathbf{Y})$ representing the unknown variables for this system, is at least equal to $\mu_{q}\left[\mathbf{U}_{\mathbf{0}, \mathbf{q}}(\mathbf{Y}), \mathbf{X}_{\mathbf{s}, \mathbf{q}}(\mathbf{Y}) \cup \mathbf{Y}_{\mathbf{0 , q}}(\mathbf{Y})\right]-$ $\rho_{q}\left[\mathbf{U}_{\mathbf{0}, \mathbf{q}}(\mathbf{Y}), \mathbf{X}_{\mathbf{s}, \mathbf{q}}(\mathbf{Y}) \cup \mathbf{Y}_{\mathbf{0}, \mathbf{q}}(\mathbf{Y})\right]$ because all the linkings between $\mathbf{U}_{\mathbf{0}, \mathbf{q}}(\mathbf{Y})$ and $\mathbf{X}_{\mathbf{s}, \mathbf{q}}(\mathbf{Y}) \cup \mathbf{Y}_{\mathbf{0}, \mathbf{q}}(\mathbf{Y})$ cover vertices included in $\mathbf{U}_{\mathbf{0 , q}}(\mathbf{Y}) \cup \mathbf{X}_{\mathbf{0 , q}}(\mathbf{Y})$. Since, in the best case, the number of independent observation equations is equal to the number of unknown variables, we 
cannot eliminate unknown variables from some equations to obtain a relation linking only $Y_{0, q}$ and $X_{s, q}$ and their derivatives.

Lemma 2 For subsystem $\left(\Sigma_{1, q}\right)$, for each $\mathbf{Y}_{\mathbf{u}}=$ $\left\{\mathbf{y}_{\mathbf{i}_{1, \mathbf{q}}}, \mathbf{y}_{\mathbf{i}_{\mathbf{2}}}, \ldots, \mathbf{y}_{\mathbf{i}_{\mathbf{k}}}\right\} \subset \mathbf{Y}_{\mathbf{1}, \mathbf{q}}(\mathbf{Y})$ such that $\rho_{q}\left[\mathbf{U}_{\mathbf{1}, \mathbf{q}}(\mathbf{Y}) \cup\right.$ $\left.\mathbf{X}_{\mathbf{s}, \mathbf{q}}(\mathbf{Y}), \mathbf{Y}_{\mathbf{u}}\right]=\operatorname{card}\left(\mathbf{U}_{\mathbf{1}, \mathbf{q}}(\mathbf{Y}) \cup \mathbf{X}_{\mathbf{s}, \mathbf{q}}(\mathbf{Y})\right)$, there exist generically a matrix $G$, a function $\varphi$ and an integer $\nu \leq$ $n_{1, q}$ such that $\left(X_{s, q}^{T}, U_{1, q}^{T}\right)^{T}=\varphi\left(Y_{u}, \dot{Y}_{u}, \ldots, Y_{u}^{(\nu)}\right)+$ $G X_{1, q}$, where $Y_{u}^{(\nu)}=\left(y_{i_{1, q}}^{(\nu)}, y_{i_{2}}^{(\nu)}, \ldots, y_{i_{k}}^{(\nu)}\right)^{T}$.

Proof: According to (Dion et al., 2003) (Theorem $4)$, for subsystem $\left(\Sigma_{1, q}\right)$, the existence of $\mathbf{Y}_{\mathbf{u}}=$ $\left\{\mathbf{y}_{\mathbf{i}_{1, \mathbf{q}}}, \mathbf{y}_{\mathbf{i}_{\mathbf{2}}}, \ldots, \mathbf{y}_{\mathbf{i}_{\mathbf{k}}}\right\} \subset \mathbf{Y}_{\mathbf{1}, \mathbf{q}}(\mathbf{Y})$ such that $\rho_{q}\left[\mathbf{U}_{\mathbf{1}, \mathbf{q}}(\mathbf{Y}) \cup\right.$ $\left.\mathbf{X}_{\mathbf{s}, \mathbf{q}}(\mathbf{Y}), \mathbf{Y}_{\mathbf{u}}\right]=\operatorname{card}\left(\mathbf{U}_{\mathbf{1}, \mathbf{q}}(\mathbf{Y}) \cup \mathbf{X}_{\mathbf{s}, \mathbf{q}}(\mathbf{Y})\right)$, implies the invertibility of $\left(\Sigma_{1, q}\right)$ using only $Y_{u}$. Thus, using the inversion algorithm of (Silverman, 1969), we can express the input of such system i.e. $U_{1, q}$ and $X_{s, q}$ in function of its state $X_{1, q}$ and its output components $Y_{u}$ and their derivatives. Thus, there exist a matrix generically $G$, a function $\varphi$ and an integer $\nu \leq n_{1, q}$ such that $\left(X_{s, q}^{T}, U_{1, q}^{T},\right)^{T}=\varphi\left(Y_{u}, \dot{Y}_{u}, \ldots, Y_{u}^{(\nu)}\right)+G X_{1, q}$, where $Y_{u}^{(\nu)}=\left(y_{i_{1, q}}^{(\nu)}, y_{i_{2}}^{(\nu)}, \ldots, y_{i_{k}}^{(\nu)}\right)^{T}$.

As a corollary of this lemma, we have that:

Lemma 3 For each $\mathbf{y}_{\mathbf{i}} \in \mathbf{Y}_{\mathbf{1}, \mathbf{q}}(\mathbf{Y})$, there exists an output algebraic relation linking it to other measurements of $\mathbf{Y}_{1, \mathbf{q}}(\mathbf{Y})$.

Proof: $\mathbf{y}_{\mathbf{i}} \notin V_{e s s, q}\left[\mathbf{U}_{\mathbf{1}, \mathbf{q}}(\mathbf{Y}) \cup \mathbf{X}_{\mathbf{s}, \mathbf{q}}(\mathbf{Y}), \mathbf{Y}_{\mathbf{1}, \mathbf{q}}(\mathbf{Y})\right] \mathrm{im}-$ plies that there exists necessarily a subset $\mathbf{Y}_{\mathbf{u}} \subset \mathbf{Y}_{\mathbf{1}, \mathbf{q}}(\mathbf{Y})$ such that $\rho_{q}\left[\mathbf{U}_{\mathbf{1}, \mathbf{q}}(\mathbf{Y}) \cup \mathbf{X}_{\mathbf{s}, \mathbf{q}}(\mathbf{Y}), \mathbf{Y}_{\mathbf{u}}\right]=\operatorname{card}\left(\mathbf{U}_{\mathbf{1}, \mathbf{q}}(\mathbf{Y}) \cup\right.$ $\left.\mathbf{X}_{\mathbf{s}, \mathbf{q}}(\mathbf{Y})\right)$. Using the previous lemma, there exist a function $\varphi$ and an integer $\nu \geq 0$ such that for mode $q$, $\left(X_{s, q}^{T}, U_{1, q}^{T}\right)^{T}=\varphi\left(Y_{u}, \dot{Y}_{u}, \ldots, Y_{u}^{(\nu)}\right)+G X_{1, q}$. Substituting this in subsystem $\left(\Sigma_{1, q}\right)$, we have:

$$
\left\{\begin{aligned}
\dot{X}_{1, q} & =\left(A_{1,1}+\left(A_{1, s}, B_{1,1}\right) G\right) X_{1, q}+\varphi_{x}\left(Y_{u}, \dot{Y}_{u}, \ldots, Y_{u}^{(\nu)}\right) \\
& \stackrel{\text { def }}{=} \tilde{A} X_{1, q}+\varphi_{x}\left(Y_{u}, \dot{Y}_{u}, \ldots, Y_{u}^{(\nu)}\right) \\
Y_{1, q} & =\left(C_{1,1}+\left(C_{1, s}, D_{1,1}\right) G\right) X_{1, q}+\varphi_{y}\left(Y_{u}, \dot{Y}_{u}, \ldots, Y_{u}^{(\nu)}\right) \\
& \stackrel{\text { def }}{=} \tilde{C} X_{1, q}+\varphi_{y}\left(Y_{u}, \dot{Y}_{u}, \ldots, Y_{u}^{(\nu)}\right)
\end{aligned}\right.
$$

Consider then that the characteristic equation of matrix $\tilde{A}$ has the form $\tilde{A}^{n_{1}}+\ldots+a_{k} \tilde{A}^{k}+\ldots+a_{0} \tilde{A}=0$, where $n_{1}=\operatorname{card}\left(\mathbf{X}_{\mathbf{1}, \mathbf{q}}(\mathbf{Y})\right)$. Multiplying this equation by $\tilde{C}_{i}$, where $\tilde{C}_{i}$ is the line of matrix $\widetilde{C}$ related to output $y_{i}$ in mode $q$ in (6), we obtain an equation on the form:

$$
\left(\widetilde{C}_{i} \tilde{A}^{n_{1}}+\ldots+a_{k} \tilde{C}_{i} \tilde{A}^{k}+\ldots+a_{0} \tilde{C}_{i} \tilde{A}\right) X_{1, q}=0
$$

Since, $y_{i}$ is an output of $\Sigma_{1, q}$, at least a term $\tilde{C}_{i} \tilde{A}^{k}$ of the latter equation is not zero and depends on $y_{i}$. Then, (7) leads to an output algebraic equation where some derivatives of $y_{i}$ intervene.

Definition 4 Consider SSLS $\left(\Sigma_{\Lambda}\right)$ associated to $\mathrm{di}$ graph $\mathcal{G}\left(\Sigma_{\Lambda}\right)$. To each vertex subset $\mathbf{V}_{\mathbf{Y}} \subseteq \mathbf{Y}$ and $q \in\{1,2\}$, we associate the integer $\beta_{q}\left(\mathbf{V}_{\mathbf{Y}}\right)$ defined as $\mu_{q}\left[\mathbf{U}_{\mathbf{0}, \mathbf{q}}\left(\mathbf{V}_{\mathbf{Y}}\right), \mathbf{X}_{\mathbf{s}, \mathbf{q}}\left(\mathbf{V}_{\mathbf{Y}}\right) \cup \mathbf{Y}_{\mathbf{0}, \mathbf{q}}\left(\mathbf{V}_{\mathbf{Y}}\right)\right]-$ $\rho_{q}\left[\mathbf{U}_{\mathbf{0 , q}}\left(\mathbf{V}_{\mathbf{Y}}\right), \mathbf{X}_{\mathbf{s}, \mathbf{q}}\left(\mathbf{V}_{\mathbf{Y}}\right) \cup \mathbf{Y}_{\mathbf{0 , q}}\left(\mathbf{V}_{\mathbf{Y}}\right)\right]$ plus the maximal number of vertices of $\mathbf{X}_{\mathbf{1}, \mathbf{q}}\left(\mathbf{V}_{\mathbf{Y}}\right) \cup \mathbf{U}_{\mathbf{1}, \mathbf{q}}\left(\mathbf{V}_{\mathbf{Y}}\right) \cup \mathbf{X}_{\mathbf{s}, \mathbf{q}}\left(\mathbf{V}_{\mathbf{Y}}\right)$ covered by a disjoint union of
- $a\left(\mathbf{U}_{\mathbf{1}, \mathbf{q}}\left(\mathbf{V}_{\mathbf{Y}}\right) \cup \mathbf{X}_{\mathbf{s}, \mathbf{q}}\left(\mathbf{V}_{\mathbf{Y}}\right)\right) \rightarrow \mathbf{Y}_{\mathbf{1}, \mathbf{q}}\left(\mathbf{V}_{\mathbf{Y}}\right)$ linking of maximal size;

- $\mathbf{Y}_{\mathbf{1}, \mathbf{q}}\left(\mathbf{V}_{\mathbf{Y}}\right)$-topped paths ;

- cycles covering only elements of $\mathbf{X}_{\mathbf{1}, \mathbf{q}}\left(\mathbf{V}_{\mathbf{Y}}\right)$.

Lemma 4 Consider SSLS $\left(\Sigma_{\Lambda}\right)$ represented by digraph $\mathcal{G}\left(\Sigma_{\Lambda, \text { lin }}\right), \beta_{q}\left(\mathbf{V}_{\mathbf{Y}}\right)$ is equal to the generic dimension of the observable subspace in the extended state and input space $\left(x^{T}(t), u^{T}(t)\right)^{T}$ for mode $q$ and restricting the measurements to the output components associated to $\mathbf{V}_{\mathbf{Y}}$.

Proof: Using results proved in (van der Woude, 2000) (Theorems 5.1 and 5.2 ) and in (van der Woude et al., 2003) (Theorem 3.6) and considering that the measurements of the system are reduced to the components associated to the elements of $\mathbf{V}_{\mathbf{Y}}$, for each mode $q$, number $\beta_{q}\left(\mathbf{V}_{\mathbf{Y}}\right)$ is equal to $n+m-g \_n_{q, i n v, z}\left(P_{q}(s)\right)$, where $g \_n_{q, i n v, z}\left(P_{q}(s)\right)$ denotes the generic number of invariant zeros of the pencil matrix $P_{q}(s)$ associated to mode $q$. Moreover, from (Trentelman et al., 2001) (Lemma 7.7), we have that $n+m-g \_n_{q, i n v, z}\left(P_{q}(s)\right)$ is also equal to the dimension of the observable subspace, i.e. the number of observable directions or independent state and input combinations, in the extended state and input space.

To achieve the discrete mode observability analysis, it is necessary to have an output algebraic relation linking some outputs and their derivatives and specific to only one of the two modes. When several output components appear in this equation, that means that for some order $k_{i}, y_{i}^{\left(k_{i}\right)}$ can be written as a linear combination of $y_{i}^{(k)}, k<k_{i}$, and of other output components with their derivatives. In this case, the intersection of observability subspace generated by $y_{i}$ and the other components is not empty. This can be characterized by the generic dimensions of such subspaces and so by $\beta_{q}(\cdot)$.

Definition 5 For each output $\mathbf{y}_{\mathbf{i}} \in \mathbf{Y}_{\mathbf{1}, \mathbf{q}}(\mathbf{Y})$, and each mode $q$, we define integer $d_{q}\left(\mathbf{y}_{\mathbf{i}}\right)=\beta_{q}(Y)-\beta_{q}\left(\mathbf{Y} \backslash\left\{\mathbf{y}_{\mathbf{i}}\right\}\right)$.

Obviously, for all $k>d_{q}\left(\mathbf{y}_{\mathbf{i}}\right)$, in mode $q, y_{i}^{(k)}$ can be written as a linear combination of $y_{i}^{(j)}, j \leq d_{q}\left(\mathbf{y}_{\mathbf{i}}\right)$, and other output components with their derivatives.

\subsection{Discrete mode observability analysis}

Using the previous settings and definitions, we analyse, hereafter, the existence of an output algebraic relation depending on the mode to achieve mode distinguishability.

Proposition $1 \operatorname{SSLS}\left(\Sigma_{\Lambda}\right)$, with two possible modes $q \in$ $\{1,2\}$, associated to digraph $\mathcal{G}\left(\Sigma_{\Lambda}\right)$ is generically discrete mode observable iff one of the following conditions is satisfied:

1.) For some $q \in\{1,2\}$, there exists a cycle $\mathcal{C}$ included in $\mathcal{E}_{0} \cup \mathcal{E}_{q}$, covering only elements of $\mathbf{X}_{\mathbf{1}, \mathbf{q}}(\mathbf{Y})$ and containing at least one edge of $\mathcal{E}_{q}$;

2.) For some $q \in\{1,2\}$, there exists a direct $\mathbf{X}_{\mathbf{s}, \mathbf{q}}(\mathbf{Y}) \cup \mathbf{U}_{\mathbf{1}, \mathbf{q}}(\mathbf{Y}) \rightarrow \mathbf{Y}_{\mathbf{1}, \mathbf{q}}(\mathbf{Y})$ path included in $\mathcal{E}_{0} \cup \mathcal{E}_{q}$ and containing at least one edge of $\mathcal{E}_{q}$;

3.) For $q \neq q^{\prime}, \mathbf{Y}_{\mathbf{1}, \mathbf{q}} \neq \mathbf{Y}_{\mathbf{1}, \mathbf{q}^{\prime}}$;

4.) For some $q \in\{1,2\}$, there exist a vertex subset $\mathbf{V}_{\mathbf{Y}} \subseteq \mathbf{Y}_{\mathbf{1}, \mathbf{q}}(\mathbf{Y})$ such that $\mathbf{V}_{\mathbf{Y}} \subseteq \mathbf{Y}_{\mathbf{1}, \mathbf{q}}\left(\mathbf{V}_{\mathbf{Y}}\right)$ and either $\mathbf{V}_{\mathbf{Y}} \nsubseteq \mathbf{Y}_{\mathbf{1}, \mathbf{q}^{\prime}}\left(\mathbf{V}_{\mathbf{Y}}\right)$ or $\beta_{q}\left(\mathbf{V}_{\mathbf{Y}}\right)>\beta_{q^{\prime}}\left(\mathbf{V}_{\mathbf{Y}}\right)$;

5.) For some $q \in\{1,2\}, \exists$ an edge $e_{\kappa} \in \mathcal{E}_{q}$ ending by 
$\mathbf{x}_{\ell} \in \mathbf{X}_{\mathbf{1}, \mathbf{q}}(\mathbf{Y})$, there exists a $\mathbf{v}_{\mathbf{P}} \rightarrow \mathbf{y}_{\mathbf{i}}$ path of length strictly greater than $d_{q}\left(\mathbf{y}_{\mathbf{i}}\right)$ which covers $e_{\kappa}$ and such that $\mathbf{x}_{\ell}$ belongs to a direct $S_{q}^{o}\left[\left\{\mathbf{v}_{\mathbf{P}}\right\}, \mathbf{Y}_{\mathbf{1}, \mathbf{q}}\right] \rightarrow \mathbf{y}_{\mathbf{i}}$ path included in $\mathcal{E}_{0} \cup \mathcal{E}_{q}$.

Proof: Sufficiency: We will prove hereafter that each of the five conditions of Proposition 1 leads to the existence of an output algebraic relation which is satisfied in only one mode and so, according to Definition 1 ensures the mode distinguishability.

Condition 1: Let us denote by $\mathbf{x}_{\mathbf{i}}$ one of the vertices of cycle $\mathcal{C}$ verifying Condition 1. Since this vertex belongs to $\mathbf{X}_{\mathbf{1}, \mathbf{q}}(\mathbf{Y})$, there exist a $\mathbf{x}_{\mathbf{i}} \rightarrow \mathbf{y}_{\mathbf{j}}$ path in $\mathcal{E}_{0} \cup \mathcal{E}_{q}$, where $\mathbf{y}_{\mathbf{j}} \in \mathbf{Y}_{\mathbf{1}, \mathbf{q}}(\mathbf{Y})$ and a subset $\mathbf{Y}_{\mathbf{u}} \subset \mathbf{Y}_{\mathbf{1}, \mathbf{q}}(\mathbf{Y}) \backslash\left\{\mathbf{y}_{\mathbf{i}}\right\}$ with $\rho_{q}\left[\mathbf{U}_{\mathbf{1}, \mathbf{q}}(\mathbf{Y}) \cup \mathbf{X}_{\mathbf{s}, \mathbf{q}}(\mathbf{Y}), \mathbf{Y}_{\mathbf{u}}\right]=\operatorname{card}\left(\mathbf{U}_{\mathbf{1}, \mathbf{q}}(\mathbf{Y}) \cup\right.$ $\left.\mathbf{X}_{\mathbf{s}, \mathbf{q}}(\mathbf{Y})\right)<\rho_{q}\left[\mathbf{U}_{\mathbf{1}, \mathbf{q}}(\mathbf{Y}) \cup \mathbf{X}_{\mathbf{s}, \mathbf{q}}(\mathbf{Y}) \cup\left\{\mathbf{x}_{\mathbf{i}}\right\}, \mathbf{Y}_{\mathbf{u}} \cup\left\{\mathbf{y}_{\mathbf{j}}\right\}\right]$. From Lemma 2 , there exist a matrix $G$, a function $\varphi$ and an integer $\nu \geq 0$ such that for mode $q,\left(X_{s, q}^{T}, U_{1, q}^{T}\right)^{T}=$ $\varphi\left(Y_{u}, \dot{Y}_{u}, \ldots, Y_{u}^{(\nu)}\right)+G X_{1, q}$. Substituting this in subsystem $\left(\Sigma_{1, q}\right)$ and using notations of (4):

$$
\left\{\begin{aligned}
\dot{X}_{1, q}= & \left(A_{1,1}+\left(A_{1, s}, B_{1,1}\right) G\right) X_{1, q}+\varphi_{x}\left(Y_{u}, \dot{Y}_{u}, \ldots, Y_{u}^{(\nu)}\right) \\
& \stackrel{\text { def }}{=} \tilde{A} X_{1, q}+\varphi_{x}\left(Y_{u}, \dot{Y}_{u}, \ldots, Y_{u}^{(\nu)}\right) \\
Y_{1, q}= & \left(C_{1,1}+\left(C_{1, s}, D_{1,1}\right) G\right) X_{1, q}+\varphi_{y}\left(Y_{u}, \dot{Y}_{u}, \ldots, Y_{u}^{(\nu)}\right) \\
& \stackrel{\text { def }}{=} \tilde{C} X_{1, q}+\varphi_{y}\left(Y_{u}, \dot{Y}_{u}, \ldots, Y_{u}^{(\nu)}\right)
\end{aligned}\right.
$$

Since the elements of $G$ are represented by the edges belonging to the $\mathbf{Y}_{\mathbf{u}}$-topped paths, they are independent from the elements of $\tilde{A}$ associated to cycle $\mathcal{C}$. Thus, the digraph representation of (6) contains also $\mathcal{C}$. Therefore, from (Reinschke, 1988) (Theorem 21.1), the characteristic equation of matrix $\tilde{A}$, has the form $\tilde{A}^{n_{1}}+\ldots+a_{k} \tilde{A}^{k}+\ldots+$ $a_{0} \tilde{A}=0$, where $n_{1}=\operatorname{card}\left(\mathbf{X}_{\mathbf{1}, \mathbf{q}}(\mathbf{Y})\right)$ and contains a term $a_{n_{1}-\bar{k}} \tilde{A}_{1,1}^{n_{1}-\bar{k}}$, where $\bar{k}$ is the length of $\mathcal{C}$ and $a_{n_{1}-\bar{k}}$ depends on the product of the weights of the edges constituting $\mathcal{C}$ and so, on a specific entry of $A_{q}$ according to the existence of an edge belonging to $\mathcal{E}_{q}$ in $\mathcal{C}$. Thus,

$$
\left(\widetilde{C}_{j} \tilde{A}^{n_{1}}+\ldots+a_{k} \tilde{C}_{j} \tilde{A}^{k}+\ldots+a_{0} \tilde{C}_{j} \tilde{A}\right) X_{1, q}=0
$$

where $\tilde{C}_{j}$ is the line of matrix $\widetilde{C}$ related to output $y_{j}$ in mode $q$ in (6) i.e. $y_{j}=\tilde{C}_{j} X_{1, q}+\varphi_{y, j}\left(Y_{u}, \dot{Y}_{u}, \ldots, Y_{u}^{(\nu)}\right)$. On the one hand, since $\mathbf{x}_{\mathbf{i}}$ belongs to a cycle and as there exists a $\mathbf{x}_{\mathbf{i}} \rightarrow \mathbf{y}_{\mathbf{j}}$ path if we denote by $\ell>0$ the length of this path, then $\forall k \geq \ell-1, \tilde{C}_{j} \tilde{A}^{k} \neq 0$. Furthermore, since $\ell+\bar{k} \leq n_{1}$, then term $a_{n_{1}-\bar{k}} \tilde{C}_{j} \tilde{A}^{n_{1}-\bar{k}} \neq 0$ and also term $\tilde{C}_{j} \tilde{A}^{k}$. On the other hand, due to dynamics (6) for all $k \geq 0 \tilde{C}_{j} \tilde{A}^{k}=y_{j}^{(k)}+\varphi_{u}\left(Y_{u}, \dot{Y}_{u}, \ldots, Y_{u}^{(\nu+k)}\right)$. Thus, (7) leads to an equation in the form:

$$
y_{j}^{\left(n_{1}+1\right)}+\ldots+a_{k} y_{j}^{(k)}+\ldots+a_{0} y_{j}+v\left(Y_{u}, \ldots, Y_{u}^{\left(2 n_{1}\right)}\right)=0
$$

Therefore, as $a_{n_{1}-\bar{k}}$ is specific to mode $q$, equation (7) leads to an output algebraic relation of the kind $\Psi_{q}\left(Y_{1, q}, \dot{Y}_{1, q}, \ldots, Y_{1, q}^{(s)}\right)=0$ parametrized by at least an element of $A_{q}$ specific to mode $q$.

Condition 2: Let denote by $\mathbf{v}_{\mathbf{i}}$ the vertex of $\mathbf{U}_{\mathbf{1}, \mathbf{q}}(\mathbf{Y}) \cup \mathbf{X}_{\mathbf{s}, \mathbf{q}}(\mathbf{Y})$ from which there is a direct
$\mathbf{X}_{\mathbf{s}, \mathbf{q}}(\mathbf{Y}) \cup \mathbf{U}_{\mathbf{1}, \mathbf{q}}(\mathbf{Y}) \rightarrow \mathbf{Y}_{\mathbf{1 , q}}(\mathbf{Y})$ path $P$ included in $\mathcal{E}_{0} \cup \mathcal{E}_{q}$ and containing at least an edge $e_{\kappa}$ of $\mathcal{E}_{q}$. Let us denote also by $\lambda_{\kappa}$ the non-zero parameter (or weight) associated to $e_{\kappa}, \mathbf{y}_{\mathbf{j}}$ the end of $P$ and $\ell$ its length. Since $\mathbf{v}_{\mathbf{i}}$ belongs to $\mathbf{U}_{\mathbf{1}, \mathbf{q}}(\mathbf{Y}) \cup \mathbf{X}_{\mathbf{s}, \mathbf{q}}(\mathbf{Y})$, it exists a subset

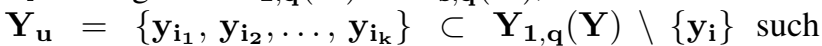
that $\rho_{q}\left[\mathbf{U}_{\mathbf{1}, \mathbf{q}}(\mathbf{Y}) \cup \mathbf{X}_{\mathbf{s}, \mathbf{q}}(\mathbf{Y}), \mathbf{Y}_{\mathbf{u}}\right]=\operatorname{card}\left(\mathbf{U}_{\mathbf{1}, \mathbf{q}}(\mathbf{Y}) \cup\right.$ $\mathbf{X}_{\mathbf{s}, \mathbf{q}}(\mathbf{Y})$ ) without using edges and vertices of $P$. From Lemma 2, there exist a matrix $G$, a function $\varphi$ and an integer $\nu \leq n_{1}$ such that the dynamics equation of subsystem $\left(\Sigma_{1, q}\right)$ is in the form (6). Since the elements of $G$ are represented by edges belonging to the $\mathbf{Y}_{\mathbf{u}}$-topped paths, they are independent from the elements of $\tilde{A}$. From the characteristic equation of matrix $\tilde{A}$, we can write an equation as (7), where also $\tilde{C}_{j}$ is the line of matrix $\widetilde{C}$ related to output $y_{j}$ in mode $q$ in (6) i.e. $y_{j}=\tilde{C}_{j} X_{1, q}+$ $\varphi_{y, j}\left(Y_{u}, \dot{Y}_{u}, \ldots, Y_{u}^{(\nu)}\right)$. Using equations of 6), we have $\tilde{C}_{j} \tilde{A}^{k} X_{1, q}=y_{j}^{(k)}-\tilde{C}_{j}\left(\varphi_{x}^{(k-1)}\left(Y_{u}, \dot{Y}_{u}, \ldots, Y_{u}{ }^{(\nu)}\right)+\right.$ $\tilde{A} \varphi_{x}^{(k-2)}\left(Y_{u}, \dot{Y}_{u}, \ldots, Y_{u}^{(\nu)}\right) \quad+\ldots+$ $\left.\tilde{A}^{k-2} \dot{\varphi}_{x}\left(Y_{u}, \dot{Y}_{u}, \ldots, Y_{u}^{(\nu)}\right)\right)-\varphi_{y, j}^{(k)}\left(Y_{u}, \dot{Y}_{u}, \ldots, Y_{u}^{(\nu)}\right)$. Since there exists a path of length $\ell$ from $\mathbf{v}_{\mathbf{i}}$ to $\mathbf{y}_{\mathbf{j}}$ containing an edge $e_{\kappa}$, term $C_{j} \tilde{A}^{\ell-1}$, for all $k \geq \ell$, is not zero and depends on the element of $A_{q}$ denoted previously $\lambda_{\kappa}$ specific to mode $q$. On the other hand, term $\varphi_{x}^{(k-\ell)}\left(Y_{u}, \dot{Y}_{u}, \ldots, Y_{u}^{(\nu)}\right)$ is not zero for all $k \geq \ell$, because there exists a path between $\mathbf{v}_{\mathbf{i}}$ and an element of $\mathbf{Y}_{\mathbf{u}}$ as by definition of $\mathbf{Y}_{\mathbf{u}} \rho_{q}\left[\mathbf{U}_{\mathbf{1}, \mathbf{q}}(\mathbf{Y}) \cup \mathbf{X}_{\mathbf{s}, \mathbf{q}}(\mathbf{Y}), \mathbf{Y}_{\mathbf{u}}\right]=$ $\operatorname{card}\left(\mathbf{U}_{\mathbf{1}, \mathbf{q}}(\mathbf{Y}) \cup \mathbf{X}_{\mathbf{s}, \mathbf{q}}(\mathbf{Y})\right)$. This implies that, $C_{j} \tilde{A}^{\ell-1} \varphi_{x}^{(k-\ell)}\left(Y_{u}, \dot{Y}_{u}, \ldots, Y_{u}^{(\nu)}\right)$ is not zero for all $k \geq \ell$ and depends on $\lambda_{\kappa}$. In particular, this implies that term $\tilde{C}_{j} \tilde{A}^{n_{1}}$, present in relation (7) is not zero and depends on $\lambda_{\kappa}$. Thus, substituting terms $\tilde{C}_{j} \tilde{A}^{k} X_{1, q}$ by $y_{j}^{(k)}-\tilde{C}_{j}\left(\varphi_{x}^{(k-1)}\left(Y_{u}, \dot{Y}_{u}, \ldots, Y_{u}{ }^{(\nu)}\right)+\right.$ $\tilde{A} \varphi_{x}^{(k-2)}\left(Y_{u}, \dot{Y}_{u}, \ldots, Y_{u}^{(\nu)}\right) \quad+\ldots+$ $\left.\tilde{A}^{k-2} \dot{\varphi}_{x}\left(Y_{u}, \dot{Y}_{u}, \ldots, Y_{u}^{(\nu)}\right)\right)-\varphi_{y, j}^{(k)}\left(Y_{u}, \dot{Y}_{u}, \ldots, Y_{u}^{(\nu)}\right)$, in equation (7), we obtain an output algebraic relation and knowing that term $C_{j} \tilde{A}^{\ell-1} \varphi_{x}^{(k-\ell)}\left(Y_{u}, \dot{Y}_{u}, \ldots, Y_{u}^{(\nu)}\right)$ is specific to mode $q$, the obtained output algebraic relation is parametrized by at least a specific element of $A_{q}$ and so allows to achieve the distiguishability.

Condition 3: This condition implies that there exists $\mathbf{y}_{\mathbf{i}} \in \mathbf{Y}_{\mathbf{1}, \mathbf{q}}(\mathbf{Y}) \backslash \mathbf{Y}_{\mathbf{1}, \mathbf{q}^{\prime}}(\mathbf{Y})$. That means, using Lemma 3 , that all the possible output algebraic relations in mode $q^{\prime}$ do not include this $y_{i}$ or its derivatives but there is an output algebraic relation including $y_{i}$ and other outputs of $\mathbf{Y}_{\mathbf{1}, \mathbf{q}}(\mathbf{Y})$ and their derivatives in mode $q$. Therefore, the latter relation is satisfied only in mode $q$ and so achieves discrete mode observability.

Condition 4: If $\mathbf{V}_{\mathbf{Y}} \nsubseteq \mathbf{Y}_{\mathbf{1}, \mathbf{q}^{\prime}}(\mathbf{Y})$, that means from Lemma 3 that there exists a relation linking the components of $\mathbf{V}_{\mathbf{Y}}=\left\{y_{i_{1}}, y_{i_{2}}, \ldots, y_{i_{\ell}}\right\}$ and their derivatives in mode $q$ but not in mode $q^{\prime}$. This achieves the discrete mode observability. Otherwise, if $\beta_{q}\left(\mathbf{V}_{\mathbf{Y}}\right)>\beta_{q^{\prime}}\left(\mathbf{V}_{\mathbf{Y}}\right)$, then the generic dimension of the observability subspace associated to $\mathbf{V}_{\mathbf{Y}}$ is greater in mode $q$ than in mode $q^{\prime}$. In this case, let us denote by $k_{i_{1}}, k_{i_{2}}, \ldots, k_{i_{\ell}}$ an $\ell$-uple of integers such that the observability subspace associated to $\mathbf{V}_{\mathbf{Y}}$ by in mode $q$ is generated by $Y_{e}=$ $\left(y_{i_{1}}, \dot{y}_{i_{1}}, \ldots, y_{i_{1}}^{\left(k_{i_{1}}\right)}, y_{i_{2}}, \dot{y}_{i_{2}} \ldots, y_{i_{\ell}}, \ldots, y_{i_{\ell}}^{\left(k_{i_{\ell}}\right)}\right)^{T} \quad$ where 


$$
\sum_{j=1}^{\ell}\left(k_{i_{j}}+1\right)=\beta_{q}\left(\mathbf{V}_{\mathbf{Y}}\right) \text {. Since, } \beta_{q^{\prime}}\left(\mathbf{V}_{\mathbf{Y}}\right)<\beta_{q}\left(\mathbf{V}_{\mathbf{Y}}\right)
$$

at least one output derivative $y_{i_{j}}^{\left(k_{i_{j}}\right)}$ can be expressed in mode $q^{\prime}$ using lower derivatives of $y_{i_{j}}$ and other elements of $Y_{e}$. This expression is valid in mode $q^{\prime}$ but not in mode $q$ and this achieves discrete mode observability.

Condition 5: The fact that, for some $q, \mathbf{y}_{\mathbf{i}}$ belongs to $\mathbf{Y}_{\mathbf{1}, \mathbf{q}}(\mathbf{Y})$ implies that there exists a vertex subset $\mathbf{Y}_{\mathbf{u}} \subseteq$ $\mathbf{Y}_{\mathbf{1}, \mathbf{q}}(\mathbf{Y}) \backslash\left\{\mathbf{y}_{\mathbf{i}}\right\}$ such that $\rho_{q}\left[\mathbf{U}_{\mathbf{1}, \mathbf{q}}(\mathbf{Y}) \cup \mathbf{X}_{\mathbf{s}, \mathbf{q}}(\mathbf{Y}), \mathbf{Y}_{\mathbf{u}}\right]=$ $\operatorname{card}\left(\mathbf{U}_{\mathbf{1}, \mathbf{q}}(\mathbf{Y}) \cup \mathbf{X}_{\mathbf{s}, \mathbf{q}}(\mathbf{Y})\right)$. This implies, from Lemma 2, that there exist a matrix $G$, a function $\varphi$ and an integer $\nu \leq n_{1}$ such that the dynamics equation of subsystem $\left(\Sigma_{1, q}\right)$ can be put on form (6). Moreover, by definition of $d_{q}\left(\mathbf{y}_{\mathbf{i}}\right)$, we have that, the dimension of the observability subspace is equal to integer $d_{q}\left(\mathbf{y}_{\mathbf{i}}\right)$ plus the dimension of the observability subspace without $y_{i}$. This means that, the $d_{q}^{t h}\left(\mathbf{y}_{\mathbf{i}}\right)$ derivative of $y_{i}$ can be expressed using the other output components and the lower derivatives of $y_{i}$. Thus, $\forall k \geq d_{q}\left(\mathbf{y}_{\mathbf{i}}\right)$, there exist a minimal subset $\tilde{\mathbf{Y}} \subseteq \mathbf{Y}_{\mathbf{1}, \mathbf{q}}(\mathbf{Y}) \backslash\left(\mathbf{Y}_{\mathbf{u}} \cup\left\{\mathbf{y}_{\mathbf{i}}\right\}\right)$, such that $y_{i}^{(k)}$ is a linear combination of the first derivatives of $y_{i}^{(s)}, s=0, \ldots, k-1$ and the all the possible derivatives (until the $n_{1}^{t h}$ the dimension of the under-determined part) of components $y_{l}$ of $\tilde{\mathbf{Y}}$. Thus, there exist some constant real parameters denoted $\alpha_{i, s}$ and $\alpha_{l, s}$ such that

$$
y_{i}^{(k)}=\sum_{s<\tilde{k_{i}}} \alpha_{i, s} y_{i}^{(s)}+\sum_{l \mid \mathbf{y}_{l} \in \tilde{\mathbf{Y}}} \sum_{s=0}^{n_{1}} \alpha_{l, s} y_{l}^{(s)}+v\left(Y_{u}, \ldots, Y_{u}^{\left(2 n_{1}\right)}\right)
$$

where $n_{1}=\operatorname{card}\left(\mathbf{X}_{\mathbf{1}, \mathbf{q}}\right)$. The aim of the rest of the proof is to show that if Condition $\mathbf{5}$ is satisfied, some of these parameters are specific to mode $q$ and this relation allows to achieve the mode distinguishability.

Using the system's equations described in (6), we can deduce that for each $s$ and $l, y_{i}^{(s)}=\tilde{C}_{i} \tilde{A}^{s} X_{1, q}+$ $f c t_{i, s}\left(Y_{u}, \ldots, Y_{u}^{\left(n_{1}+s\right)}\right)$ and $y_{l}^{(s)}=\tilde{C}_{l} \tilde{A}^{s} X_{1, q}+$ $f c t_{l, s}\left(Y_{u}, \ldots, Y_{u}^{\left(n_{1}+s\right)}\right)$, where $\tilde{C}_{i}$ (resp. $\tilde{C}_{l}$ ) is the row of matrix $\tilde{C}$ corresponding to output $y_{i}$ (resp. $y_{l}$ ). Substituting the latter equalities in (9), all functions of $Y_{u}$ and its derivatives will simplify as they are independent from the considered elements of $X_{1, q}$ and (9) can be written as

$$
\tilde{C}_{i} \widetilde{A}^{k} X_{1, q}=\sum_{s<d_{q}\left(\mathbf{y}_{\mathbf{i}}\right)} \alpha_{i, s} \tilde{C}_{i} \widetilde{A}^{s} X_{1, q}+\sum_{\mathbf{y}_{l} \in \tilde{\mathbf{Y}}} \sum_{s=0}^{n_{1}} \alpha_{l, s} \tilde{C}_{l} \tilde{A}^{s} X_{1, q}
$$

Since subset $\tilde{\mathbf{Y}}$ is minimal i.e. $\forall \mathbf{y}_{\mathbf{j}} \in \tilde{\mathbf{Y}}, \beta_{q}\left(\tilde{\mathbf{Y}} \cup\left\{\mathbf{y}_{\mathbf{i}}\right\} \cup\right.$ $\left.\left.\mathbf{Y}_{\mathbf{u}}\right) \backslash\left\{\mathbf{y}_{\mathbf{j}}\right\}\right)-\beta_{q}\left(\mathbf{Y}_{\mathbf{u}} \cup \tilde{\mathbf{Y}} \backslash\left\{\mathbf{y}_{\mathbf{j}}\right\}\right)>\widetilde{k_{i}}$, then in relation (10), all the components of $\tilde{\mathbf{Y}}$ intervene. Let us denote by $\mathbf{v}_{\mathbf{P}}=\mathbf{x}_{\mathbf{j}}$ the begin vertex of the so-called path $P$ satisfying Condition $\mathbf{5}$ (i.e. $P$ is a $\mathbf{y}_{\mathbf{i}}$-topped path of length $k+1$ strictly greater than $d_{q}\left(\mathbf{y}_{\mathbf{i}}\right)$ and covers $\left.e_{s}\right)$ and $\mathrm{e}_{j}$ the $j^{\text {th }}$ Euclidean vector. As this relation is valid whatever the state vector $X_{1, q}$, we can remove it and by multiplying relation (10) in the right by $\mathrm{e}_{j}$, we obtain:

$$
\tilde{C}_{i} \widetilde{A}^{k} \mathrm{e}_{j}=\left(\sum_{s<d_{q}\left(\mathbf{y}_{\mathbf{i}}\right)} \alpha_{i, s} \tilde{C}_{i} \widetilde{A}^{s}+\sum_{\mathbf{y}_{l} \in \tilde{\mathbf{Y}}} \sum_{s=0}^{n_{1}} \alpha_{l, s} \tilde{C}_{l} \tilde{A}^{s}\right) \mathrm{e}_{j}
$$

where each non-zero component of $\tilde{C}_{l} \tilde{A}^{s}$ is associated to the paths arriving to $\mathbf{y}_{l} \in \tilde{\mathbf{Y}}$ of length $s+1$. Since all the $\left\{\mathbf{x}_{\mathbf{j}}\right\} \rightarrow \tilde{\mathbf{Y}} \cup\left\{\mathbf{y}_{\mathbf{i}}\right\}$ paths starting from $\mathbf{x}_{\mathbf{j}}$ cover, by definition, $S_{q}^{o}\left[\left\{\mathbf{x}_{\mathbf{j}}\right\}, \tilde{\mathbf{Y}} \cup\left\{\mathbf{y}_{\mathbf{i}}\right\}\right] \stackrel{\text { def }}{=}\left\{\mathbf{x}_{\mathbf{r}}\right\}$, then there exist $k_{r}$ and $k^{\prime}$ such that $k_{r}+k^{\prime}=k$ and $\tilde{C}_{i} \tilde{A}^{k} \mathrm{e}_{j}=\tilde{C}_{i} A^{k_{r}} \Delta_{r} \tilde{A}^{k^{\prime}} \mathrm{e}_{j}$ where $\Delta_{r}$ is a diagonal matrix having only one nonzero element $\Delta_{r}(r, r)=1$. We can do the same reasoning for each term $\tilde{C}_{l} \tilde{A}^{s} \mathrm{e}_{j}$ and so there exist $s_{r}$ and $s^{\prime}$ such that $s_{r}^{\prime}+s^{\prime}=s$ and $\tilde{C}_{l} \tilde{A}^{s} \mathrm{e}_{j}=\tilde{C}_{l} \tilde{A}^{s_{r}} \Delta_{r} \tilde{A}^{s^{\prime}} \mathrm{e}_{j}$. The fact that end vertex of $e_{\kappa}, \mathbf{x}_{\ell}$ belongs to a direct $S_{q}^{o}\left[\left\{\mathbf{x}_{\mathbf{j}}\right\}, \mathbf{Y}_{\mathbf{1}, \mathbf{q}}\right] \rightarrow \mathbf{y}_{\mathbf{i}}$ path implies that specific edge $e_{\kappa} \in \mathcal{E}_{q}$ belongs to a $S_{q}^{o}\left[\mathbf{v}_{\mathbf{P}}, \tilde{\mathbf{Y}} \cup\left\{\mathbf{y}_{\mathbf{i}}\right\}\right] \rightarrow \tilde{\mathbf{Y}} \cup\left\{\mathbf{y}_{\mathbf{i}}\right\}$ path. This means that edge $e_{\kappa}$ appears in only some of $S_{q}^{o}\left[\mathbf{v}_{\mathbf{P}}, \tilde{\mathbf{Y}} \cup\left\{\mathbf{y}_{\mathbf{i}}\right\}\right] \rightarrow \tilde{\mathbf{Y}} \cup\left\{\mathbf{y}_{\mathbf{i}}\right\}$ paths. Thus, some, but not all, terms $\tilde{C}_{i} A^{k_{r}}$ and $\tilde{C}_{l} \tilde{A}^{s_{r}}$ contain the non-zero parameter corresponding to edge $e_{\kappa}$, specific to mode $q$. Denoting by $C_{r}=\mathrm{e}_{r}^{T}$, where $\mathrm{e}_{r}$ is the $r^{t h}$ Euclidean vector, we have that $\tilde{C}_{i} \tilde{A}^{k} \mathrm{e}_{j}=\tilde{C}_{i} A^{k_{r}} \Delta_{r} \tilde{A}^{k^{\prime}} \mathrm{e}_{j}=\alpha^{\prime} C_{r} \tilde{A}^{k^{\prime}} \mathrm{e}_{j}$ and $\tilde{C}_{l} \tilde{A}^{s} \mathrm{e}_{j}=\tilde{C}_{l} \tilde{A}^{s_{r}} \Delta_{r} \tilde{A}^{s^{\prime}} \mathrm{e}_{j}=\alpha_{l, s}^{\prime} C_{r} \tilde{A}^{s^{\prime}} \mathrm{e}_{j}$, where $\alpha_{l, s}^{\prime} \stackrel{\text { def }}{=}\left(\tilde{C}_{l} \tilde{A}^{s_{r}} \Delta_{r}\right)\left(C_{r} C_{r}^{T}\right)^{-1}=\left(\tilde{C}_{l} \tilde{A}^{s_{r}} \Delta_{r}\right)$. Thus, after substitution of the previous terms in relation (11),

$$
\begin{aligned}
\alpha^{\prime} C_{r} \widetilde{A}^{k^{\prime}} \mathrm{e}_{j}= & \left(\sum_{s_{r} \leq s<d_{q}\left(\mathbf{y}_{\mathbf{i}}\right)} \alpha_{i, s}^{\prime} \alpha_{i, s} C_{r} \widetilde{A}^{s-s_{r}}+\right. \\
& \left.\sum_{l \mid \mathbf{y}_{l} \in \tilde{\mathbf{Y}}} \sum_{s=s_{r}}^{n_{1}} \alpha_{l, s}^{\prime} \alpha_{l, s} C_{r} \tilde{A}^{s-s_{r}}\right) \mathrm{e}_{j}
\end{aligned}
$$

where some, but not all coefficients $\alpha^{\prime}$ and $\alpha_{l, s}^{\prime}$ depend on the weight of $e_{\kappa}$. This weight cannot be factorized and simplified because all the coefficients do not depend on it (some $S_{q}^{o}\left[\mathbf{v}_{\mathbf{P}}, \tilde{\mathbf{Y}} \cup\left\{\mathbf{y}_{\mathbf{i}}\right\}\right] \rightarrow \tilde{\mathbf{Y}} \cup\left\{\mathbf{y}_{\mathbf{i}}\right\}$ paths do not contain edge $e_{\kappa}$ ). Therefore, equality (12) is valid only if some of the coefficients $\alpha, \alpha_{i, s}$ and $\alpha_{l, s}$ depend also on the weight $\lambda_{\kappa}$ of $e_{\kappa}$. Thus, by means of equation (9) containing coefficients $\alpha_{i, s}$ and $\alpha_{l, s}$, we obtain an output algebraic relation depending on $\lambda_{\kappa}$ and satisfied only when the discrete mode variable is equal to $q$.

Necessity: Firstly, from Lemma 1, there cannot exist an output algebraic relation linking the output components of the under-determined subsystem $\left(\Sigma_{0, q}\right)$. So, only the edges in subsystem $\left(\Sigma_{1, q}\right)$ can lead to an output algebraic relation (between outputs $\mathbf{Y}_{\mathbf{1}, \mathbf{q}}(\mathbf{Y})$ and their derivatives) useful to deduce the discrete mode. In this case, some outputs have to be used to invert $\left(\Sigma_{1, q}\right)$ in order to eliminate from the output equation all the unknown inputs and so to use all the possible derivatives of the other outputs in an output algebraic equation. It is necessary then to consider a subset $\mathbf{Y}_{\mathbf{u}} \subseteq \mathbf{Y}_{\mathbf{1}, \mathbf{q}}(\mathbf{Y})$ such that $\rho_{q}\left[\mathbf{U}_{\mathbf{1}, \mathbf{q}}(\mathbf{Y}) \cup\right.$ $\left.\mathbf{X}_{\mathbf{s}, \mathbf{q}}(\mathbf{Y}), \mathbf{Y}_{\mathbf{u}}\right]=\operatorname{card}\left(\mathbf{U}_{\mathbf{1}, \mathbf{q}}(\mathbf{Y}) \cup \mathbf{X}_{\mathbf{s}, \mathbf{q}}(\mathbf{Y})\right)$. Indeed, from Lemma 2 and Theorem 4 of (Dion et al., 2003), only in such a case the system is left-invertible and so there exist integer $\nu \geq 0$, matrix $G$ and function $\varphi$ such that $\left(X_{s, q}^{T}, U_{1, q}^{T}\right)^{T}=\varphi\left(Y_{u}, \dot{Y}_{u}, \ldots, Y_{u}^{(\nu)}\right)+G X_{1, q}$. This quantity can then be substituted in subsystem $\left(\Sigma_{1, q}\right)$ for mode $q$, to obtain an equation of form (6).

When Conditions $\mathbf{1}$ and $\mathbf{2}$ are not satisfied, it is not possible to obtain an output algebraic relation achieving mode distinguishability from the characteristic equation of any 
matrix $\tilde{A}$ of (6) related to any choice of $\mathbf{Y}_{\mathbf{u}}$, when this equation has the same degree for the two modes. It is the case when Condition $\mathbf{4}$ is not satisfied. Moreover, when Condition $\mathbf{2}$ is not satisfied, for any possible choice of $Y_{u}, \varphi_{y}\left(Y_{u}, \dot{Y}_{u}, \ldots, Y_{u}^{(\nu)}\right)$ and $\varphi_{x}\left(Y_{u}, \dot{Y}_{u}, \ldots, Y_{u}^{(\nu)}\right)$ do not depend on any specific element of $A_{q}, B_{q}, C_{q}$ or $D_{q}$, $q \in\{1,2\}$. Furthermore, any output relation can be put on the form (9), for some integer $k$, output component $\mathbf{y}_{\mathbf{i}}$ and some set of output components $\widetilde{\mathbf{Y}}$ which do not include $\mathbf{y}_{\mathbf{i}}$ and are disjoint from $\mathbf{Y}_{\mathbf{u}}$. Moreover, when Conditions $\mathbf{3}$ and $\mathbf{4}$ are not satisfied, the output components implicated in any of these expressions and their derivatives degrees are the same for modes 1 and 2 . Thus, the only way to have a difference between the two output algebraic equations respectively is that there exists at least one coefficient $\alpha_{i, s}$ or $\alpha_{l, s}$ of Equation (9) characterizing specifically a parameter specific only to one of the two modes' model. Without loss of generality, we can assume that $\widetilde{\mathbf{Y}}$ is minimal i.e. in the output algebraic relations and so all the components of $\tilde{\mathbf{Y}}$ appear. If it is not the case, we remove them from $\widetilde{\mathbf{Y}}$. Relation (9) implies that the observable subspace obtained using outputs $\left\{\mathbf{y}_{\mathbf{i}}\right\} \cup \mathbf{Y}_{\mathbf{u}}$ and the one generated by $\tilde{\mathbf{Y}} \cup \mathbf{Y}_{\mathbf{u}}$ have non zero intersection. Defining $\widetilde{k}_{i}=\beta_{q}\left(\tilde{\mathbf{Y}} \cup\left\{\mathbf{y}_{\mathbf{i}}\right\} \cup \mathbf{Y}_{\mathbf{u}}\right)-\beta_{q}\left(\tilde{\mathbf{Y}} \cup \mathbf{Y}_{\mathbf{u}}\right) \geq d_{q}\left(\mathbf{y}_{\mathbf{i}}\right)$, relation (9) cannot be satisfied for some $k \leq \widetilde{k_{i}}-1$. Thus, in output algebraic relation (9), $k \geq \widetilde{k}_{i}$. Furthermore, since $\tilde{\mathbf{Y}}$ is minimal, we cannot express $y_{i}^{\left(\tilde{k_{i}}\right)}$ using only a part of $\tilde{Y}$ i.e. $\forall \mathbf{y}_{\mathbf{j}} \in \tilde{\mathbf{Y}}$. To guarantee discrete mode observability, there must exist at least a $\mathbf{y}_{\mathbf{i}}$-topped path $P$, of length greater or equal to $\widetilde{k}_{i}+1$ and so of length strictly greater than $d_{q}\left(\mathbf{y}_{\mathbf{i}}\right)$, associated to this relation. Let us denote by $\mathbf{x}_{\mathbf{j}}$ the begin vertex of $P$ (it cannot be a $\mathbf{U}_{\mathbf{1}, \mathbf{q}}(\mathbf{Y}) \cup \mathbf{X}_{\mathbf{s}, \mathbf{q}}(\mathbf{Y}) \rightarrow \mathbf{Y}_{\mathbf{1}, \mathbf{q}}(\mathbf{Y})$ path when Condition $\mathbf{2}$ is not satisfied) and $\mathrm{e}_{j}$ the $j^{\text {th }} \mathrm{Eu}-$ clidean vector. When Condition 2 is not satisfied, in Equation (9), $v\left(Y_{u}, \dot{Y}_{u}, \ldots, Y_{u}^{(n)}\right)$ does not depend on any element specific of $A_{q}, B_{q}, C_{q}$ or $D_{q}(q \in\{1,2\})$. Then, equation (9), can be written as relation (11), where $\tilde{C}_{l} \tilde{A}^{s}$ is associated $\mathbf{y}_{l}$-topped paths, $\mathbf{y}_{l} \in \tilde{\mathbf{Y}}$ of length $s+1$. All the $\left\{\mathbf{x}_{\mathbf{j}}\right\} \rightarrow \tilde{\mathbf{Y}} \cup\left\{\mathbf{y}_{\mathbf{i}}\right\}$ paths cover $\mathbf{x}_{\mathbf{r}}=S_{q}^{o}\left[\mathbf{x}_{\mathbf{j}}, \tilde{\mathbf{Y}} \cup\left\{\mathbf{y}_{\mathbf{i}}\right\}\right]$. So, if for each $\mathrm{x}_{\ell}$ end vertex of a specific edge belonging to $P, \mathbf{x}_{\ell}$ does not belong to any direct $S_{q}^{o}\left[\left\{\mathbf{v}_{\mathbf{P}}\right\}, \mathbf{Y}_{\mathbf{1}, \mathbf{q}}\right] \rightarrow \mathbf{y}_{\mathbf{i}}$ path, then all the paths starting from $\mathbf{x}_{\mathbf{r}}$ to $\mathbf{Y}$ contain only edges of $\mathcal{E}_{0}$. Then according to the same arguments as previously (in the part of the proof devoted to the sufficiency of Condition 5), we can write an equation similar to (12) with the same notations, where $\alpha^{\prime}$ and $\alpha_{l, s^{\prime}}^{\prime}$ depend only on the entries related to the edges of $\mathcal{E}_{0}$ when Condition $\mathbf{5}$ is not satisfied. Equation (12) implies that there exists an output algebraic relation with the use of only virtual output $\tilde{C}_{r} x$ in addition to $Y_{u}$. This relation can be due to the characteristic equation of matrix $\widetilde{A}$ which includes only the terms related to cycles in the digraph. When Condition 1 is not satisfied, there is no edge of $\mathcal{E}_{q}, q \in\{1,2\}$ in any cycle. Thus, as all $\alpha^{\prime}$ and $\alpha_{l, s^{\prime}}^{\prime}$ depend only on the entries related to the edges of $\mathcal{E}_{0}$, it is also the case for coefficients $\alpha_{i, s}$ and $\alpha_{l, s}$ in equation (9). So, all the existing relations of the form (9) do not contain terms of $A_{q}$, $B_{q}, C_{q}$ or $D_{q}(q \in\{1,2\})$. We can do the same reasoning for all $\mathbf{Y}_{\mathbf{u}} \subseteq \mathbf{Y}_{\mathbf{1}, \mathbf{q}}(\mathbf{Y})$ s.t. $\rho_{q}\left[\mathbf{U}_{\mathbf{1}, \mathbf{q}}(\mathbf{Y}) \cup \mathbf{X}_{\mathbf{s}, \mathbf{q}}(\mathbf{Y}), \mathbf{Y}_{\mathbf{u}}\right]$ $=\operatorname{card}\left(\mathbf{U}_{\mathbf{1}, \mathbf{q}}(\mathbf{Y}) \cup \mathbf{X}_{\mathbf{s}, \mathbf{q}}(\mathbf{Y})\right)$.

Comments and interpretation: To establish the discrete mode observability, we first search in the graph the edges specific to one mode and we interest to their position w.r.t. the output vertices. If there is a specific edge belonging to any cycle in $\left(\Sigma_{1, q}\right)$ then the distinguishability is possible (first condition). If a specific edge belong to any input $\rightarrow$ output path in $\left(\Sigma_{1, q}\right)$ then the distinguishability is possible (second condition). If a specific edge allows to modify the output subdivision $\mathbf{Y}_{\mathbf{0}} / \mathbf{Y}_{\mathbf{1}}$ (third condition) or to modify observability subspace of any output measurements set (fourth condition), then the distinguishability is possible. Finally, if a specific edge belongs to an output rooted path including a state vertex which can be linked independently to other outputs, then the distinguishability is possible (fifth condition). If no condition is satisfied, then the two modes are sufficiently similar or their differences are not observable from the measurements and so they are not distinguishable.

An intuitive structural interpretation of the conditions and what they entail can be also provided, knowing that an output algebraic relation can exist only in the over-determined part of a system and that any output belonging to this overdetermined part belongs to at least one output algebraic equation:

- Condition 1 means that there exists an output combination with a dynamics depending on the discrete mode variable but not from the unknown input.

- Condition 2 means that an observable input-output behaviour depends on the discrete mode variable. This dependence is structural when the relative degree of the considered input changes else it is parametric.

- Condition 3 means that the under-determined part of the system changes according to the discrete mode variable. Thus, there exists an output algebraic relation including a particular output $y_{i}$ in a mode while all the possible output algebraic equations in the other mode do not include this output component.

- Condition 4 means that the dimension of the observability subspace associated to some output components depends on the discrete mode variable. This is equivalent to say that the observability index of some output components changes and so the modes are distinguishable from their observability characteristics. This implies that there exists an output algebraic relation between several outputs of the system and whose order depends on the discrete mode.

- Condition 5 means that there exists an output algebraic relation between several outputs of the system and that this relation contains some parameters depending on the discrete mode.

To summarize, the conditions provided in Proposition 1 can be classified into two categories. The first one includes Conditions 3, 4, 2 when the subdivision of the system for the two modes are different and a part of Condition $\mathbf{1}$. In this case, the two modes can be said structurally distinguishable. The distinguishability here depends on the structure of the systems associated to each mode and not on the parameters' values. In the case of Condition $\mathbf{1}$, when the cycle exists in a mode and not in the other one, there is then at least an additional relation differentiating the two modes and so the distinguishability is here also structural. When 
only Condition $\mathbf{5}$, the distinguishability is purely parametric.

The main differences with the formulation given in (Boukhobza and Hamelin, 2011) of the discrete mode observability conditions lie in three points:

- Condition 3 of (Boukhobza and Hamelin, 2011) has been split in two conditions here Condition 3 and Condition 4. This is done for computational reasons. Indeed, Condition 3 of (Boukhobza and Hamelin, 2011) is stated as "For some $q \in\{1,2\}$, there exist a vertex $\mathbf{y}_{\mathbf{i}} \in \mathbf{Y}_{\mathbf{1}, \mathbf{q}}(\mathbf{Y})$ and disjoint vertex subsets $\tilde{\mathbf{Y}} \subseteq \mathbf{Y}_{\mathbf{1}, \mathbf{q}}(\mathbf{Y}) \backslash\left\{\mathbf{y}_{\mathbf{i}}\right\}$ (this subset can be empty), $\mathbf{Y}_{\mathbf{u}} \subseteq \mathbf{Y}_{\mathbf{1}, \mathbf{q}}(\mathbf{Y}) \backslash\left\{\mathbf{y}_{\mathbf{i}}\right\}$ such that:..." three conditions must be verified. This implies a combinatorial search to check a property on all the output vertices of $\mathbf{Y}_{\mathbf{1 , q}}(\mathbf{Y})$ but also on all the possible subsets $\tilde{\mathbf{Y}}$ and $\mathbf{Y}_{\mathbf{u}}$. In the present paper, Condition $\mathbf{3}$ does not necessitate any computation and for Condition 4, we check a test on only one output vertex subset $\mathbf{V}_{\mathbf{Y}}$. On the one hand, this is computationally easier and on the other hand, globally only two conditions must be verified on $\mathbf{V}_{\mathbf{Y}}$, knowing that one of them $\left(\mathbf{V}_{\mathbf{Y}} \subseteq \mathbf{Y}_{\mathbf{1}, \mathbf{q}}\left(\mathbf{V}_{\mathbf{Y}}\right)\right)$ can be expressed easily using a linking condition.

- Condition 4 of (Boukhobza and Hamelin, 2011) is substituted in Proposition 1 by Condition 5. In (Boukhobza and Hamelin, 2011), checking Condition 4 implies, as previously, to test 5 properties, on all possible groups constituted by an output vertex, a path and two disjoint output vertices. In the present paper, checking Condition $\mathbf{5}$ is equivalent to check a condition on the position of a specific edge w.r.t. its distance to an output vertex. We do that by working on a simplified graph where are removed all the edges which do not belong to a sufficiently long path. Condition 5 necessitates the computation of, at most, $n$ output separators. - Due to the changes in the formulation of the discrete mode conditions, as we will see below, the sensor location study to recover the discrete mode observability is possible because it can be centred on the position of specific edges w.r.t. output vertices in the digraph. With conditions given in (Boukhobza and Hamelin, 2011), this is not possible, because the two last ones depend on a group constituted of at least three parts: a single output vertex and two subsets of output vertices. Thus, when adding a sensor, we must consider all the cases where each of these groups changes. In the conditions given in Proposition 1, we must check a property for at most one output vertex or one output vertex subset.

Remark 2 When all the inputs are known, according to Remark $1, \Sigma_{1, q}, q \in 1,2$ will contain all the input vertices and all the state vertices which are connected to the outputs. That means that, the subdivision of the system do not give an under-determined part. Consequently, the specific edges belonging to any $Y$-topped path may be useful to establish the discrete mode observability. This can be illustrated in Example 1 where edge $\left(\mathbf{x}_{\mathbf{6}}, \mathbf{x}_{\mathbf{7}}\right)$ is specific but as it belongs to $\Sigma_{0, q}$ part, $q \in 1,2$, it cannot be used to generate an output algebraic relation specific to mode 1 or mode 2 . But, if input $u_{2}$ is known (or measured), this edge will belong to an input-output path in $\Sigma_{1, q}, q \in 1,2$, Condition 2 will be satisfied and the weight of this edge participates to an output algebraic relation specific to mode 1 or mode 2 .

Note that, when all the input are measured, the Proposition is applicable, by adding in the system output measurements on all the inputs i.e. output vertices and edges linking each known input vertex to its associated measurement vertex.

Example 1 Continued It is easy to see that Condition $\mathbf{1}$ is satisfied while Condition $\mathbf{2}$ is not. Indeed, cycle $\mathbf{x}_{\mathbf{4}} \rightarrow \mathbf{x}_{\mathbf{4}}$ contains a specific edge related to mode 1 and $\mathbf{x}_{\mathbf{4}} \in \mathbf{X}_{\mathbf{1 , 1}}$, then Condition $\mathbf{1}$ is satisfied and the system is discrete mode observable. Moreover, $\mathbf{U}_{\mathbf{1 , 1}}=\mathbf{U}_{\mathbf{1 , 2}}=\emptyset$ and $\mathbf{X}_{\mathbf{s}, \mathbf{1}}=\mathbf{X}_{\mathbf{s}, \mathbf{2}}=\left\{\mathbf{x}_{\mathbf{8}}\right\}$ but there is no direct path including specific edges between $\mathbf{X}_{\mathbf{s}, \mathbf{1}}$ or $\mathbf{X}_{\mathbf{s}, \mathbf{2}}$ and $\mathbf{Y}$, so Condition 2 cannot be satisfied.

Let us check Condition $\mathbf{5}$. There is only one edge $\left(\mathbf{x}_{\mathbf{2}}, \mathbf{x}_{\mathbf{1}}\right)$ specific to mode 1 and this edge can belong only to $\mathbf{y}_{\mathbf{1}}-$ topped paths. So, let us compute $d_{1}\left(\mathbf{y}_{1}\right): \beta_{1}(\mathbf{Y})=9$ and $\beta_{1}\left(\mathbf{Y} \backslash\left\{\mathbf{y}_{\mathbf{1}}\right\}\right)=8$. Thus, $d_{1}\left(\mathbf{y}_{\mathbf{1}}\right)=1 . \operatorname{Pred}_{\mathbf{1}}\left(\left\{\mathbf{x}_{\mathbf{1}}\right\}\right)=$ $\left\{\mathbf{x}_{\mathbf{2}}, \mathbf{x}_{\mathbf{3}}\right\}$ and we have $S_{1}^{o}\left(\mathbf{x}_{\mathbf{2}}, \mathbf{Y}_{\mathbf{1}, \mathbf{1}}\right)=S_{1}^{o}\left(\mathbf{x}_{\mathbf{3}}, \mathbf{Y}_{\mathbf{1}, \mathbf{1}}\right)=$ $\left\{\mathbf{x}_{2}\right\} . \mathbf{x}_{1}$ belongs to a direct $\left\{\mathbf{x}_{2}\right\} \rightarrow \mathbf{y}_{1}$ path included in $\mathcal{E}_{0} \cup \mathcal{E}_{1}$. Let us search now a $\mathbf{y}_{1}$-topped path $P$ whose length is greater or equal to $d_{1}\left(\mathbf{y}_{1}\right)+1=2$ and it includes edge $\left(\mathbf{x}_{2}, \mathbf{x}_{1}\right)$. We can choose $P=\mathbf{x}_{2} \rightarrow \mathbf{x}_{1} \rightarrow \mathbf{y}_{1}$ with $\mathbf{v}_{\mathbf{P}}=\mathbf{x}_{\mathbf{2}}$ and so, Condition $\mathbf{5}$ is then satisfied. This graphic condition characterizes output algebraic relation $\frac{\dot{y}_{1}}{\lambda_{7} \lambda_{11}}-\frac{\dot{y}_{2}}{\lambda_{8} \lambda_{14}}=0$ depending on $\lambda_{11}$ and $\lambda_{14}$ and specific to mode 1 only. This relation is not satisfied for mode 2 because, in this case $\dot{y}_{1}$ is identically zero. Relation $\dot{y}_{1}=0$ is specific to mode 2 even if there is no specific coefficient to mode 2 appearing because here Condition $\mathbf{4}$ is satisfied: or in other terms, $\beta_{1}\left(\left\{\mathbf{y}_{\mathbf{1}}\right\}\right)=3 \neq \beta_{2}\left(\left\{\mathbf{y}_{\mathbf{1}}\right\}\right)=1$. This traduces that in mode $2, \dot{y}_{1}=0$ while it is not the case in mode 1 .

To handle a ically specified tnumero a specific realization of the structured system with numerically specified parameters, we only apply the previous generic results. In the case of the specific system given at the end of Example 1 , we can conclude to the discrete mode observability for almost any unknown input and initial state because the following output relations allow to distinguish the mode: $\dot{y}_{1}=0$ which is valid in mode in mode $2, \dot{y}_{1}-\frac{\dot{y}_{2}}{2}=0$ and $\dot{y}_{3}=-3 y_{3}+y_{2}-y_{4}$ which are valid only in mode 1 .

Note that sometimes (it is not the case for this example), the generic algebraic relation which allows the distinguishability, have some singularities w.r.t. the parameter values. In this case, for the specific realizations presenting these singularities, the generic result is not applicable.

\subsection{Additional sensor location to recover the mode ob- servability}

When none of the conditions of Proposition 1 is satisfied, we propose hereafter a strategy in order to precise as finely as possible the additional sensors' locations allowing to distinguish the two discrete modes. This problem is more complicated than a classical observability problem (Commault et al., 2008; Boukhobza and Hamelin, 2009) or fault diagnosability one (Commault and Dion, 2007) because of the objective is not to detect or to observe a state variable or an input variable but to detect a change in a dynamics. Knowing that this information is supported only by the edges, it is pertinent to concentrate our reasoning particularly on the ones which are not common to the two modes. Choosing one of these specific edges, denoted $e_{s}=\left(\mathbf{v}_{\mathbf{s}}, \mathbf{v}_{\mathbf{s}}^{\prime}\right) \in \mathcal{E}_{q}$, we will treat all the situations, according to its position in the digraph. To obtain all the additional sensors' locations 
ensuring the discrete mode observability, it is then sufficient to roll out the following procedure for all the edges which are not common to the two modes. In the sequel, $\mathbf{Y}_{\text {add }}$ denotes the set of additional sensors. Obviously, the added sensors are considered to be common to the two modes. Indeed, it is not realistic to add sensors only active during a certain mode, especially when the system is not discrete mode observable.

1) $e_{s}=\left(\mathbf{v}_{\mathbf{s}}, \mathbf{v}_{\mathbf{s}}^{\prime}\right)$ belongs to a cycle but included $\Sigma_{0, q}$ : In this case, to satisfy to Condition $\mathbf{1}$ it is necessary to add a sensor in order to make $\mathbf{v}_{\mathbf{s}}^{\prime}$ belonging to $\Sigma_{1, q}$. It does not matter if $\mathbf{v}_{\mathbf{s}}$ (when different from $\mathbf{v}_{\mathbf{s}}^{\prime}$ ) still belongs to $\Sigma_{0, q}$ after the additional sensor placement, because in this case, $\mathbf{v}_{\mathbf{s}}$ will belong to $\mathbf{X}_{\mathbf{s}, \mathbf{q}}(\mathbf{Y})$ and Condition 2 will be satisfied. Thus, the objective is to have $\mathbf{v}_{\mathbf{s}}^{\prime} \in \mathbf{X}_{\mathbf{1}, \mathbf{q}}\left(\mathbf{Y} \cup \mathbf{Y}_{\text {add }}\right)$. Therefore, let us denote by $\mathbf{S}_{\mathbf{u}, \mathbf{q}}=$ $\mathbf{S}_{\mathbf{q}}^{\mathbf{i}}(\mathbf{U}, \mathbf{Y})$, it is necessary (but not always sufficient) to place a sensor to measure any vertex covered by any direct $\mathbf{S}_{\mathbf{u}, \mathbf{q}} \cup\left\{\mathbf{v}_{\ell}\right\} \rightarrow \mathbf{S}_{\mathbf{q}}^{\mathbf{i}}\left(\mathbf{S}_{\mathbf{u}, \mathbf{q}} \cup\left\{\mathbf{v}_{\ell}\right\}, \mathbf{Y}\right)$ path and strictly before $\mathbf{S}_{\mathbf{q}}^{\mathbf{i}}\left(\mathbf{S}_{\mathbf{u}, \mathbf{q}} \cup\left\{\mathbf{v}_{\ell}\right\}, \mathbf{Y}\right)$, where $\mathbf{v}_{\ell}$ is any vertex belonging to the cycle. If, after adding a sensor, the cycle is not in $\Sigma_{1, q}$, we must repeat the placement above considering the new sensor set until obtaining $\mathbf{v}_{\mathbf{s}}^{\prime} \in \mathbf{X}_{\mathbf{1}, \mathbf{q}}\left(\mathbf{Y} \cup \mathbf{Y}_{\text {add }}\right)$. The maximal number of additional sensors to make this inclusion true is $\operatorname{card}\left(\mathbf{S}_{\mathbf{u}, \mathbf{q}} \cup\left\{\mathbf{v}_{\mathbf{s}}^{\prime}\right\}\right)-\rho_{q}\left[\mathbf{S}_{\mathbf{u}, \mathbf{q}} \cup\left\{\mathbf{v}_{\mathbf{s}}^{\prime}\right\}, \mathbf{Y}\right]$.

Example 2 Consider now the SSLS represented by the digraph depicted in Figure 3.

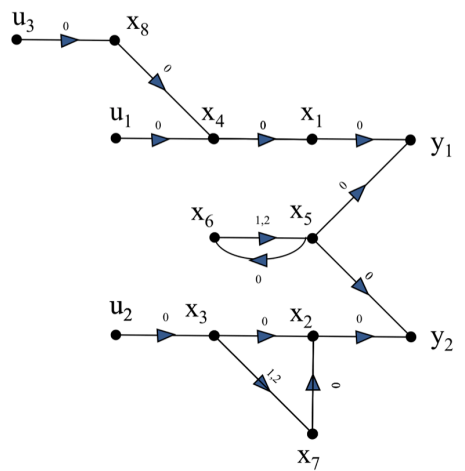

Figure 3. Digraph associated to the system of Example 2

For this example, the differences between the two modes lie in two edges: $\left(\mathbf{x}_{\mathbf{5}}, \mathbf{x}_{\mathbf{6}}\right)$ and $\left(\mathbf{x}_{\mathbf{3}}, \mathbf{x}_{\mathbf{7}}\right)$. All the state, input and output vertices belong to $\Sigma_{0, q}$ for the two modes because of $\rho_{1}[\mathbf{U}, \mathbf{Y}]=\rho_{2}[\mathbf{U}, \mathbf{Y}]=2=\operatorname{card}(\mathbf{Y})$. Thus, $c y$ cle $\mathrm{x}_{5} \rightarrow \mathrm{x}_{\mathbf{6}} \rightarrow \mathrm{x}_{5}$ containing a specific edge to mode 1 is included also in $\Sigma_{0,1}$. To satisfy Condition 1, we must add a sensor. Here, $\mathbf{S}_{\mathbf{u}, \mathbf{1}}=\left\{\mathbf{x}_{\mathbf{4}}, \mathbf{u}_{\mathbf{2}}\right\}$ and $\mathbf{S}_{\mathbf{1}}^{\mathbf{i}}\left(\mathbf{S}_{\mathbf{u}, \mathbf{1}} \cup\left\{\mathbf{x}_{\mathbf{6}}\right\}, \mathbf{Y}\right)=$ $\mathbf{S}_{\mathbf{1}}^{\mathbf{i}}\left(\mathbf{S}_{\mathbf{u}, \mathbf{1}} \cup\left\{\mathbf{x}_{\mathbf{5}}\right\}, \mathbf{Y}\right)=\left\{\mathbf{y}_{\mathbf{1}}, \mathbf{y}_{\mathbf{2}}\right\}$. Therefore, an additional sensor measuring any vertex on any $\left\{\mathbf{x}_{4}, \mathbf{u}_{2}, \mathbf{x}_{5}\right\} \rightarrow$ $\left\{\mathbf{y}_{1}, \mathbf{y}_{2}\right\}$ path or $\left\{\mathbf{x}_{4}, \mathbf{u}_{2}, \mathbf{x}_{\mathbf{6}}\right\} \rightarrow\left\{\mathbf{y}_{1}, \mathbf{y}_{2}\right\}$ path i.e. any component in $\left\{\mathbf{x}_{1}, \mathbf{x}_{\mathbf{2}}, \mathbf{x}_{\mathbf{3}}, \mathbf{x}_{\mathbf{4}}, \mathbf{x}_{\mathbf{5}}, \mathbf{x}_{\mathbf{6}}, \mathbf{x}_{\mathbf{7}}, \mathbf{u}_{\mathbf{2}}\right\}$ is necessary and here sufficient to ensure the discrete mode observability. Note that, we find $u_{2}$ in the previous subset. Indeed, as it is highlighted in Remark 2 when input $u_{2}$ is known, then the under-determined part of the system includes more vertices and edges and this makes the discrete mode-observability possible.
2) $e_{s}=\left(\mathbf{v}_{\mathbf{s}}, \mathbf{v}_{\mathbf{s}}^{\prime}\right)$ belongs to an $U$-rooted simple path but included $\Sigma_{0, q}$ : In this case, to satisfy to Condition 2 , it is necessary and sufficient to add a sensor in order to make $\mathbf{v}_{\mathbf{s}}$ either in $\mathbf{X}_{\mathbf{s}, \mathbf{q}}\left(\mathbf{Y} \cup \mathbf{Y}_{\text {add }}\right)$ or in $\mathbf{X}_{\mathbf{1}, \mathbf{q}}\left(\mathbf{Y} \cup \mathbf{Y}_{\text {add }}\right)$. Therefore, let us denote by $\mathbf{S}_{\mathbf{u}, \mathbf{q}}=\mathbf{S}_{\mathbf{q}}^{\mathbf{i}}(\mathbf{U}, \mathbf{Y})$, it is necessary and sufficient to place a sensor to measure $\mathbf{v}_{\mathbf{s}}$ or any vertex covered by any direct $\mathbf{S}_{\mathbf{u}, \mathbf{q}} \cup\left\{\mathbf{v}_{\mathbf{s}}^{\prime}\right\} \rightarrow \mathbf{S}_{\mathbf{q}}^{\mathbf{i}}\left(\mathbf{S}_{\mathbf{u}, \mathbf{q}} \cup\left\{\mathbf{v}_{\mathbf{s}}^{\prime}\right\}, \mathbf{Y}\right)$ path and strictly before $\mathbf{S}_{\mathbf{q}}^{\mathbf{i}}\left(\mathbf{S}_{\mathbf{u}, \mathbf{q}} \cup\left\{\mathbf{v}_{\mathbf{s}}^{\prime}\right\}, \mathbf{Y}\right)$.

Example 3 Consider again the SSLS represented by the digraph depicted in Figure 3. Specific edge to mode $2\left(\mathbf{x}_{\mathbf{3}}, \mathbf{x}_{\mathbf{7}}\right)$ is included in $\Sigma_{0,1}$. To satisfy Condition 2 , we must add a sensor in order to make $\mathrm{x}_{3}$ either in $\mathbf{X}_{\mathbf{s}, \mathbf{2}}\left(\mathbf{Y} \cup \mathbf{Y}_{\text {add }}\right)$ or in $\mathbf{X}_{\mathbf{1 , 2}}\left(\mathbf{Y} \cup \mathbf{Y}_{\text {add }}\right)$. Since $\mathbf{x}_{\mathbf{3}} \in V_{\text {ess }, 2}[\mathbf{U}, \mathbf{Y}]$, we can make it included as well in $\mathbf{X}_{\mathbf{s}, \mathbf{2}}\left(\mathbf{Y} \cup \mathbf{Y}_{\text {add }}\right)$ or in $\mathbf{X}_{\mathbf{1 , 2}}\left(\mathbf{Y} \cup \mathbf{Y}_{\text {add }}\right)$. Here, $\mathbf{S}_{\mathbf{u}, \mathbf{2}}=$ $\left\{\mathbf{x}_{\mathbf{4}}, \mathbf{u}_{\mathbf{2}}\right\}$ and $\mathbf{S}_{\mathbf{1}}^{\mathbf{i}}\left(\mathbf{S}_{\mathbf{u}, 2} \cup\left\{\mathbf{x}_{\mathbf{7}}\right\}, \mathbf{Y}\right)=\left\{\mathbf{x}_{\mathbf{2}}, \mathbf{x}_{\mathbf{4}}\right\}$. Therefore, to satisfy Condition 2, it is necessary and sufficient to measure $\left\{\mathbf{u}_{\mathbf{2}}, \mathbf{x}_{\mathbf{3}}, \mathbf{x}_{\mathbf{7}}\right\}$. Note that, we find $u_{2}$ in the previous subset. Indeed, as it is highlighted in Remark 2 when input $u_{2}$ is known, then the under-determined part of the system includes more vertices and edges and this makes the mode-observability possible.

Note that, by this additional sensor location, dedicated to recovering Condition 2, we can satisfy in the same time Condition 3 since these conditions are intricately linked.

3) $e_{s}=\left(\mathbf{v}_{\mathbf{s}}, \mathbf{v}_{\mathbf{s}}^{\prime}\right)$ is exclusive to one mode, not covered

neither by a cycle nor by an $U$-rooted path: In this case, the predecessors of $\mathbf{v}_{\mathbf{s}}$ do not include an input vertex. In addition to this, as the added sensor corresponds to a vertex and a common edge, it is not possible to satisfy, with any additional sensor located anywhere, Condition 3. Thus, we interest here only to recovering Condition 4 and this can be possible only if $\left(\mathbf{v}_{\mathbf{s}}, \mathbf{v}_{\mathbf{s}}^{\prime}\right)$ does not belong to $\mathcal{E}_{0}$ (is exclusive to one mode). Let us denote by $\mathbf{V}_{\mathbf{q}, \text { Obs }}$ the set of all observable input and state components, which is equal to $\left\{\mathbf{v} \in \mathbf{X} \cup \mathbf{U}, \beta_{q}(\mathbf{Y} \cup\{\mathbf{v}\})=\beta_{q}(\mathbf{Y})\right\}$ (Boukhobza, 2010). To satisfy condition $\mathbf{4}$, it is necessary and sufficient to place a sensor to take measures on the following set $\left\{\mathbf{v}_{\mathbf{s}}^{\prime}\right\} \cup\left\{\mathbf{x}_{\mathbf{j}}\right.$ successor of $\mathbf{v}_{\mathbf{s}}^{\prime}$ such that the maximal length path arriving to $\mathbf{x}_{\mathbf{j}}$ covers necessarily $\left.e_{s}\right\}$. This set can be unfortunately empty. If it is not the case, the new output allows to construct a set of outputs $\mathbf{V}_{\mathbf{Y}}$, which has not the same observability subspace dimension in mode $q$ and in mode $q^{\prime}$. Indeed, the added measurement is sensitive only in mode $q$ to $v_{s}$ representing the most distant unmeasurable state vertex for the added sensor.

Example 4 Consider the SSLS represented by the digraph depicted in Figure 4.

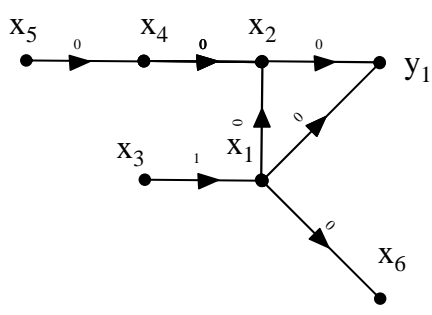

Figure 4. Digraph of the system of Example 4

For this example, without unknown inputs, all the state and output vertices belong obviously to $\Sigma_{1, q}$. But there cannot 
exist an output algebraic relation since there is only one output (Conditions 3 and 5 cannot be satisfied), no cycle (Condition 1 cannot be satisfied), no input (Condition 2 cannot be satisfied) and Condition 4 is not satisfied. Since there exists an exclusive edge $\left(\mathbf{x}_{3}, \mathbf{x}_{1}\right)$ related to mode 1 , we can satisfy Condition 4 by adding a sensor. The set of successors of $\mathbf{x}_{1}$ are $\left\{\mathbf{x}_{2}, \mathbf{x}_{\mathbf{6}}, \mathbf{y}_{1}\right\}$ and $\mathbf{V}_{\mathbf{1}, \mathrm{Obs}}=\emptyset$. We can place a sensor on $\mathbf{x}_{1}$ or on $\mathbf{x}_{6}$ since the maximal length path arriving to these vertices covers necessarily $\left(\mathbf{x}_{3}, \mathbf{x}_{1}\right)$. It is not the case for $\mathbf{x}_{2}$ since there is a maximal path arriving to $\mathrm{x}_{2}$ and starting in $\mathrm{x}_{5}$ and dos not cover $\left(\mathrm{x}_{3}, \mathrm{x}_{1}\right)$. Thus, adding a sensor on $\mathrm{x}_{1}$ or on $\mathrm{x}_{6}$ allows to satisfy Condition 4 and to recover the discrete mode observability.

4) $e_{s}=\left(\mathbf{v}_{\mathbf{s}}, \mathbf{v}_{\mathbf{s}}^{\prime}\right)$ does not belong neither to a cycle nor to an $U$-rooted simple path and the previous

step does not succeed: In this last case, the aim is to add some sensors in order to recover Condition 5. To do so, we proceed in two steps. The objective of the first step is to ensure $\mathbf{v}_{\mathbf{s}}$ belongs to a direct $S_{q}^{o}\left[\operatorname{Pred}_{\mathbf{q}}\left(\left\{\mathbf{v}_{\mathbf{s}}\right\}\right), \mathbf{Y}_{\mathbf{1 , \mathbf { q }}}\right] \rightarrow \mathbf{Y}_{\mathbf{1}, \mathbf{q}}$ path included in $\mathcal{E}_{0} \cup \mathcal{E}_{q}$. The objective of the second step is to ensure that, for at least one output $\mathbf{y}_{\mathbf{i}}$ successor of $\mathbf{v}_{\mathbf{s}}^{\prime}$, we have that $d_{q}\left(\mathbf{y}_{\mathbf{i}}\right)$ is less or equal than the maximal distance from $\mathbf{v}_{\mathbf{s}}^{\prime}$ to $\mathbf{y}_{\mathbf{i}}$.

- Step 1 : If $\mathbf{v}_{\mathbf{s}}$ does not belong to a direct $S_{q}^{o}\left[\operatorname{Pred}_{\mathbf{q}}\left(\left\{\mathbf{v}_{\mathbf{s}}\right\}\right), \mathbf{Y}_{\mathbf{1}, \mathbf{q}}\right] \rightarrow \mathbf{y}_{\mathbf{i}}$ path included in $\mathcal{E}_{0} \cup \mathcal{E}_{q}$, then it is necessary and sufficient to place a sensor to measure $\mathbf{v}_{\mathbf{s}}$ or any vertex covered by any direct $\operatorname{Pred}_{\mathbf{q}}\left(\left\{\mathbf{v}_{\mathbf{s}}\right\}\right) \rightarrow \mathbf{S}_{\mathbf{q}}^{\mathbf{i}}\left(\operatorname{Pred}_{\mathbf{q}}\left(\left\{\mathbf{v}_{\mathbf{s}}\right\}\right), \mathbf{Y}\right)$ path.

- Step 2 : If after Step 1 above, Condition 5. is not satisfied, then we propose an iterative additional sensor location procedure:

\section{While Condition 5. is not satisfied}

$\rightarrow$ choose $\mathbf{y}_{\mathbf{i}}$ one of the output components successors of $\mathbf{v}_{\mathbf{s}}^{\prime}$

$\rightarrow$ place an additional sensor on any predecessor of $\mathbf{y}_{\mathbf{i}}$ which is not in $V_{q, O b s}$ and from which the maximal distance to $\mathbf{y}_{\mathbf{i}}$ is less or equal than the one from $\mathbf{v}_{\mathbf{s}}^{\prime}$ to $\mathbf{y}_{\mathbf{i}}$.

The algorithm above leads, when we consider always the same output component successor of $\mathbf{v}_{\mathbf{s}}^{\prime}, \mathbf{y}_{\mathbf{i}}$ to the decrease of $d_{q}\left(\mathbf{y}_{\mathbf{i}}\right)$ until attaining a value less than the distance from $\mathbf{v}_{\mathbf{s}}^{\prime}$ to $\mathbf{y}_{\mathbf{i}}$. In this case, Condition $\mathbf{5}$. will be satisfied.

Example 5 Consider the SSLS represented by the digraph depicted in Figure 5.

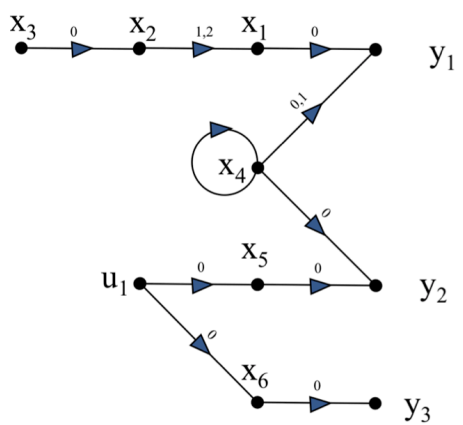

Figure 5. Digraph of the system of Example 5
We have that $\forall q \in\{1,2\}, \mathbf{X}_{\mathbf{0}, \mathbf{q}}(\mathbf{Y})=\mathbf{U}_{\mathbf{0}, \mathbf{q}}(\mathbf{Y})=$ $\mathbf{X}_{\mathbf{s}, \mathbf{q}}(\mathbf{Y})=\mathbf{Y}_{\mathbf{0}, \mathbf{q}}(\mathbf{Y})=\emptyset, \mathbf{U}_{\mathbf{1}, \mathbf{q}}(\mathbf{Y})=\left\{\mathbf{u}_{\mathbf{1}}\right\}, \mathbf{X}_{\mathbf{1}, \mathbf{q}}(\mathbf{Y})=$ $\mathbf{X}$ and $\mathbf{Y}_{\mathbf{1}, \mathbf{q}}(\mathbf{Y})=\mathbf{Y}$. Furthermore, $\forall q \in\{1,2\}$, $\beta_{q}(\mathbf{Y})=6, \beta_{q}\left(\left\{\mathbf{y}_{\mathbf{2}}, \mathbf{y}_{\mathbf{3}}\right\}\right)=3, \beta_{q}\left(\left\{\mathbf{y}_{\mathbf{1}}, \mathbf{y}_{\mathbf{3}}\right\}\right)=5$ and $\beta_{q}\left(\left\{\mathbf{y}_{\mathbf{1}}, \mathbf{y}_{\mathbf{2}}\right\}\right)=5$.

It is easy to see that the three first conditions of Proposition 1 are not satisfied. Moreover, since there is no exclusive edge, Condition 4 cannot be satisfied. The only specific edge is $\left(\mathbf{x}_{\mathbf{4}}, \mathbf{y}_{\mathbf{1}}\right)$ and has as output successor only $\mathbf{y}_{\mathbf{1}}$. When we take $\mathbf{y}_{\mathbf{i}}=\mathbf{y}_{\mathbf{1}}$ we have for both $q=1$ and $q=2$, $d_{q}\left(\mathbf{y}_{1}\right)=3$ and there is no $\mathbf{y}_{\mathbf{1}}$-topped path with a length greater than $d_{q}\left(\mathbf{y}_{1}\right)+1=4$. Thus, Condition 5 is also not satisfied and this system is not discrete mode observable. In order to recover Condition 5, we must add at least a sensor. First, note that here $\mathbf{v}_{\mathbf{P}}$ of Proposition 1, can be only $\mathbf{x}_{\mathbf{4}} . S_{1}^{0}\left[\left\{\mathbf{x}_{\mathbf{4}}\right\}, \mathbf{Y}_{\mathbf{1}, \mathbf{1}}\right]=\left\{\mathbf{x}_{\mathbf{4}}\right\}$ and we have that $\mathbf{y}_{\mathbf{1}}$ belongs to a direct $\left.\left\{\mathbf{x}_{\mathbf{4}}\right\} \rightarrow \mathbf{y}_{\mathbf{1}}\right]$ path. Therefore, the first step is not necessary. We go directly to Step 2: here $\mathbf{y}_{\mathbf{i}}=\mathbf{y}_{\mathbf{1}}$ and $V_{1, O b s}=\left\{\mathbf{x}_{2}, \mathbf{x}_{\mathbf{3}}, \mathbf{x}_{\mathbf{6}}, \mathbf{u}_{1}\right\}$. Vertices $\mathbf{x}_{\mathbf{1}}$ and $\mathbf{x}_{\mathbf{4}}$ are the only ones in te previous list from which there exists a path having a length less or equal to the ones from $\mathbf{x}_{4}$ to $\mathbf{y}_{1}$. Thus, we must measure $\mathbf{x}_{1}$ or $\mathbf{x}_{4}$.

Assume that we measure $\mathrm{x}_{1}$. In this case, the system will not be discrete mode observable since $d_{1}\left(\mathbf{y}_{1}\right)=1$ and as the path between $\mathbf{x}_{4}$ and $\mathbf{y}_{1}$ is also of length 1 . Thus, we must measure also $\mathbf{x}_{\mathbf{4}}$. In this case, $d_{1}\left(\mathbf{y}_{1}\right)=0$, Condition 5 will be satisfied. Note that instead measuring $\mathbf{x}_{4}$, we can measure also $\mathbf{x}_{\mathbf{5}}$ even if this vertex is not related to $\mathbf{y}_{1}$, it allows also to obtain $d_{1}\left(\mathbf{y}_{1}\right)=1$. In fact, our procedure leads always to a solution but is not exhaustive for this last procedure stage.

Note that, when we deal with a specific realization of a SSLS with numerically specified parameters, we can obviously apply the proposed method above in order to add sensors for recovering the discrete mode observability. After choosing the additional sensors, we must verify, as it is done for Example 1 that the obtained algebraic relations useful to establish the distinguishability presents no singularities. If it is the case, then we must eliminate the useless sensor(s) and add a new other one using the procedure above. We do that until we recover the discrete mode observability of the considered specific realization. If there exist a difference between the two modes in the system's dynamics, after a finite number of iterations the provided procedure will always allow to recover the discrete mode observability.

\section{Conclusion}

In this paper, we propose a graph-theoretic tool to analyse generic mode discrete mode observability for switching structured linear systems with unknown inputs. More precisely, we provide new necessary and sufficient conditions to ensure the generic discrete mode observability of switching structured linear systems. Next, when these observability conditions are not satisfied, we study the location of additional sensors in order to recover the generic discrete mode observability. Our approach uses classical programming techniques and is free from numerical difficulties. This makes our proposed method well-suited to treat large scale systems. 


\section{References}

Babaali, M. \& Pappas, G. J., (2005). Observability of switched linear systems in continuous time. In M. Morari and L. Thiele, Editors, Hybrid Systems: Computation and Control, volume 3414 of Lecture Notes in Computer Science, 103-117. Springer Berlin / Heidelberg.

Boukhobza, T. (2010). Partial state and input observability recovering by additional sensor implementation: a graph-theoretic approach International Journal of Systems Science, 41(11):1281 - 1291.

Boukhobza, T., \& Hamelin, F. (2009). State and input observability recovering by additional sensor implementation: a graph-theoretic approach. Automatica, 45(7), 1737-1742.

Boukhobza, T., \& Hamelin, F. (2011). Observability of switching structured linear systems with unknown input: a graph-theoretic approach. Automatica, 47(2), 395-402.

Boukhobza, T., Hamelin, F. \& Martinez-Martinez, S. (2007). State and input observability for structured linear systems: A graph-theoretic approach. Automatica, 43(7), 1204-1210.

Commault, C. \& Dion, J-M. (2007). Sensor location for diagnosis in linear Systems: a structural analysis. IEEE Transactions on Automatic Control, 52(2), 155-169.

Commault, C., Dion, J-M., \& Trinh, D. H. (2008). Observability preservation under sensor failure. IEEE Transactions on Automatic Control, 53(6), 1554-1559.

De Santis, E., Di Benedetto, M. D. \& Pola, G. (2006). Observability of internal variables in interconnected switching systems. Proceedings of the $45^{\text {th }}$ IEEE Conference on Decision and Control, 4121-4126, U.S.A.

De Santis, E., Di Benedetto, M. D. \& Pola, G. (2009). A structural approach to detectability for a class of hybrid systems. Automatica, 45(5), 1202-1206.

Dion, J-M., Commault, C., \& Van der Woude, J. (2003). Generic properties and control of linear structured systems: A survey. Automatica, 39(7), 1125-1144.

Pina, L. \& Botto, A. (2007). Simultaneous state and input estimation of hybrid systems with unknown inputs. Automatica, 42(5),755-762.

Reinschke, K. J. (1988). Multivariable Control. A Graph Theoretic Approach. Springer-Verlag. New York, U.S.A.

Silverman, L. M. (1969). Inversion of multivariable linear systems. IEEE Transactions on Automatic Control, AC-14(3), 270-276.

Trentelman, H. L., Stoorvogel, A. A. \& Hautus, M. (2001). Control Theory for Linear Systems. Springer, London, U.K.

van der Woude, J. W. (2000). The generic number of invariant zeros of a structured linear system. SIAM Journal of Control and Optimization, $38(1), 1-21$.

J. W. van der Woude, C. Commault, and J. M. Dion, Zero orders and dimensions of some invariant subspaces in linear structured systems. Mathematics of Control, Signals and Systems, 16 (2-3), 225-237.

Vidal, R., Chiuso, A., Soatto, S. \& Sastry, S. (2003). Observability of linear hybrid systems. In O. Maler and A. Pnueli, Editors, Hybrid Systems: Computation and Control, volume 2623 of Lecture Notes in Computer Science, 526-539. Springer Berlin / Heidelberg.

Vu, L. \& Liberzon, D. (2006). On invertibility of switched linear systems. Proceedings of the $45^{\text {th }}$ IEEE Conference on Decision and Control, 4081-4086, U.S.A. 